\title{
Wide-Area All-Optical Neurophysiology in Acute Brain Slices
}

\author{
(1)Samouil L. Farhi, ${ }^{1 * ~(1) V i c e n t e ~ J . ~ P a r o t, ~}{ }^{2,9 *}$ Abhinav Grama, ${ }^{3,4}$ (Masahito Yamagata, ${ }^{3,4}$ @Ahmed S. Abdelfattah, ${ }^{10}$ \\ (DYoav Adam, ${ }^{6}$ (DShan Lou, ${ }^{6}$ Jeong Jun Kim, ${ }^{6}$ (D) Robert E. Campbell, ${ }^{10}$ David D. Cox, ${ }^{3,4,5}$ and (DAdam E. Cohen ${ }^{6,7,8}$ \\ ${ }^{1}$ Chemical Biology Program, ${ }^{2}$ Biophysics Program, ${ }^{3}$ Department of Molecular and Cellular Biology, ${ }^{4}$ Center for Brain Science, ${ }^{5}$ J. A. Paulson School of \\ Engineering and Applied Sciences, ${ }^{6}$ Department of Chemistry and Chemical Biology, ${ }^{7}$ Department of Physics, ${ }^{8}$ Howard Hughes Medical Institute, Harvard \\ University, Cambridge, Massachusetts 02138, ${ }^{9}$ Division of Health Science and Technology, Massachusetts Institute of Technology, Cambridge, \\ Massachusetts 02139, and ${ }^{10}$ Department of Chemistry, University of Alberta, Edmonton, Alberta T6G 2R3, Canada
}

Optical tools for simultaneous perturbation and measurement of neural activity open the possibility of mapping neural function over wide areas of brain tissue. However, spectral overlap of actuators and reporters presents a challenge for their simultaneous use, and optical scattering and out-of-focus fluorescence in tissue degrade resolution. To minimize optical crosstalk, we combined an optimized variant $(\mathrm{eTsChR})$ of the most blue-shifted channelrhodopsin reported to-date with a nuclear-localized red-shifted $\mathrm{Ca}^{2+}$ indicator, $^{2}$ H2B-jRGEC01a. To perform wide-area optically sectioned imaging in tissue, we designed a structured illumination technique that uses Hadamard matrices to encode spatial information. By combining these molecular and optical approaches we made wide-area functional maps in acute brain slices from mice of both sexes. The maps spanned cortex and striatum and probed the effects of antiepileptic drugs on neural excitability and the effects of AMPA and NMDA receptor blockers on functional connectivity. Together, these tools provide a powerful capability for wide-area mapping of neuronal excitability and functional connectivity in acute brain slices.

Key words: brain slice; calcium imaging; microscopy; optogenetics

\section{Significance Statement}

A new technique for simultaneous optogenetic stimulation and calcium imaging across wide areas of brain slice enables highthroughput mapping of neuronal excitability and synaptic transmission.

\section{Introduction}

All-optical neurophysiology (AON), simultaneous optical stimulation and optical readout of neural activity, provides a promising approach to mapping neural excitability and functional connectivity across wide regions of brain tissue (Emiliani et al., 2015; Ronzitti et al., 2017). Recent advances in two-photon (2P) calcium imaging AON in vivo have enabled measurement of neuro-

Received Jan. 20, 2019; revised March 15, 2019; accepted April 1, 2019.

Author contributions: S.L.F., V.J.P., J.J.K., R.E.C., D.D.C., and A.E.C. designed research; S.L.F., V.J.P., A.G., A.S.A., Y.A.,S.L., and J.J.K. performed research; S.L.F., V.J.P., A.G., M.Y., A.S.A., Y.A., S.L., and J.J.K. contributed unpublished reagents/analytic tools; S.L.F., V.J.P., J.J.K., and A.E.C. analyzed data; S.L.F., V.J.P., and A.E.C. wrote the paper.

This work was supported by the Howard Hughes Medical Institute, by an NSF Graduate Research Fellowship to S.L.F, by a Becas Chile scholarship to V.J.P., and work in David Cox's laboratory was supported by IARPA (contract D16PC00002) and the Mind Brain Behavior Faculty Award of Harvard. Constructs will be made available on Addgene. Code for Hadamard pattern generation and image reconstruction, as well as raw data examples are linked in Materials and Methods. We thank Vaibhav Joshi, Katherine Williams, and Melinda Lee for technical assistance, Bernardo Sabatini for Rbp4-Cre mice, Christopher Werley for assistance with the microscope design, Daryl Lim for providing HiLo reconstruction code, and Joshua Sanes for support for the cloning of H2B-jRGECO1a.

Conflict of interest: A.E.C. and V.J.P. have filed a patent application on Hadamard microscopy. A.E.C. is a cofounder of Q-State Biosciences. The remaining authors declare no competing financial interests.

*S.L.F. and V.J.P. contributed equally to this work.

Correspondence should be addressed to Adam E. Cohen at cohen@chemistry.harvard.edu.

A. S. Abdelfattah's present address: Janelia Farm Research Campus, Ashburn, VA 20147.

https://doi.org/10.1523/JNEUROSCI.0168-19.2019

Copyright $\odot 2019$ the authors nal population activity while stimulating or inhibiting up to $\sim 100$ near-surface neurons in small cortical regions (Ronzitti et al., 2017; Shemesh et al., 2017; Mardinly et al., 2018). However, most of the intact rodent brain remains inaccessible to optical microscopy, and one would ideally like to perform AON simultaneously on many thousands of neurons across multiple brain regions to map spatial variations in function or to detect rare subpopulations.

Acute brain slices in principle enable wide-area optical mapping across any brain region. Although slicing cuts many long-range connections, the procedure is commonly used to investigate the molecular makeup, electrophysiological properties, and local microcircuitry of the component neurons (Ting et al., 2018). Wide-area AON in acute slices would enable several types of new applications. Pharmacological studies with tool compounds could probe the distribution and functional roles of receptors or channels in the tissue, e.g., to characterize the nature of synaptic connections between specific brain regions; or to discover cell populations that express a receptor for an orphan ligand (Harnett et al., 2013; Blot and Barbour, 2014; Jiang et al., 2015). Tests with candidate drugs could probe the distribution and functional consequences of drug action. Further potential applications include probing the response of brain tissue to physical or chemical perturbations, e.g., to map responses to changes in nutrients, hormones, oxygen, or temperature. In all these 
applications, a large field-of-view (FOV) for simultaneous largearea imaging is essential because the sample response might adapt or degrade, and physiology may vary as a function of time postperturbation.

Brain slices typically show little spontaneous activity and obviously lack sensory inputs, so optical mapping in brain slices requires a means to evoke activity. Optogenetic stimulation can directly evoke activity in the measured neurons, or can activate axon terminals, even when the axons have been severed from the cell bodies, to evoke postsynaptic responses (Petreanu et al., 2007). Optical readouts of evoked response could reveal the spatial structure of intrinsic neuronal excitability, of functional connectivity, or of local microcircuit dynamics and molecular or physical influences thereon.

The optical requirements of wide-area $\mathrm{AON}$ in brain slice differ from in vivo, suggesting that a distinct approach could be warranted. In brain slice there is a benefit to having a very wide FOV to probe many neurons and brain regions simultaneously. Optical sectioning is important to distinguish in-focus cells from background, but imaging deep $(>100 \mu \mathrm{m})$ into the preparation is less important than in vivo because the plane of the slice can expose any brain structure of interest. One may stimulate many thousands of cells simultaneously, a task beyond the capabilities of current $2 \mathrm{P}$ stimulation techniques. If one treats cells as units, the spatial resolution must be sufficient to resolve single cells, but need not resolve fine subcellular structures. Time resolution must be

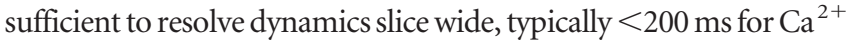
imaging. These factors, discussed in detail here, suggest that onephoton (1P) stimulation and imaging may be preferable over the $2 \mathrm{P}$ approaches which have been optimized for in vivo use. To achieve $1 \mathrm{P}$ $\mathrm{AON}$ in brain slice one must (1) identify an actuator/reporter pair with good photostability and minimal optical crosstalk under $1 \mathrm{P}$ illumination, and (2) implement a $1 \mathrm{P}$ optically sectioned wide-area imaging scheme. Here we combine molecular and optical engineering to address these challenges.

Red-shifted channelrhodopsins have been combined with a GCaMP $\mathrm{Ca}^{2+}$ indicator for $2 \mathrm{P} \mathrm{AON}$ in vivo (Rickgauer et al., 2014; Packer et al., 2015; Carrillo-Reid et al., 2016), but 1P GCaMP excitation causes spurious channelrhodopsin excitation. Lower optical crosstalk is achieved by pairing a blue-shifted channelrhodopsin with a red-shifted reporter (Hochbaum et al., 2014). Red genetically encoded $\mathrm{Ca}^{2+}$ indicators (RGECIs) now offer good sensitivity, but their combination with optogenetic stimulation has been hampered by blue-light induced photoswitching of the mApple-based chromophores used in the most sensitive RGECIs (Akerboom et al., 2013; Inoue et al., 2015; Dana et al., 2016). Furthermore, blue channelrhodopsins such as ChR2(H134R) retain some excitation at the yellow (561 nm) wavelengths used to excite RGECIs, introducing crosstalk of the imaging light into the stimulation channel. A truly orthogonal $1 \mathrm{P}$ actuator/RGECI reporter pair has not previously been reported.

TsChR, derived from Tetraselmis striata (Klapoetke et al., 2014), is the most blue-shifted channelrhodopsin reported todate, but its initial characterization yielded a poor photocurrent. To our knowledge, TsChR has not previously been used in any optogenetic experiments. Here we show that a version with improved trafficking, eTsChR, drives robust spiking in cultured neurons and in tissue. Combination of eTsChR with a nuclearlocalized red-shifted $\mathrm{Ca}^{2+}$ reporter, H2B-jRGECOla, achieved $1 \mathrm{P} A O N$ in cultured neurons and in slice. The blue light used to activate the eTsChR was dim enough to avoid jRGECOla photoswitching, and the yellow light used to excite jRGECOla did not spuriously activate the eTsChR.
On the imaging front, $1 \mathrm{P}$ structured illumination microscopy (SIM) techniques can achieve optical sectioning in tissue (Mertz, 2011). We developed a generalized SIM technique based on Hadamard-Walsh encoding and implemented it in a mesoscope imaging system. Hadamard microscopy provides better rejection of out-of-plane fluorescence than do other SIM techniques and offers the ability to make systematic tradeoffs between background rejection and time resolution.

By applying 1P optogenetic stimulation and Hadamard microscopy to acute slices expressing eTsChR and H2B-jRGECO1a, we obtained simultaneous functional characterization of $>6000$ neurons, spread over a region $2.3 \times 2.3 \mathrm{~mm}$ with $5.6 \mathrm{~Hz}$ time resolution. Maps of optically induced activity highlighted distinct cortical layers, which otherwise appeared homogeneous in their fluorescence. We used the AON system to map with cellular resolution the effects of anti-epileptic drugs on neural excitability, and to study cortico-cortico and cortico-striatal functional connectivity. Finally, we show that with an improvement to the algorithm based on compressed sensing, the imaging speed can be increased to $33 \mathrm{~Hz}$ (Parot et al., 2019). The combined molecular and optical tools provide a powerful system for wide-area investigations of neural function in brain tissue.

\section{Materials and Methods}

\section{DNA constructs}

R-CaMP2 was a gift from Haruhiko Bito. TsChR was a gift from Ed Boyden. jRGECO1a and jRCAMP1a were obtained from Addgene (plasmids 61563 and 61562). All RGECIs were cloned between the BamHI and EcoRI sites of the backbone from FCK-Arch-GFP (Addgene plasmid 22217) for expression in cultured neurons and for lentiviral production. For photophysical characterization, RGECIs were also cloned into an analog of the FCK vector replacing the CaMKII $\alpha$ promoter with a CAG promoter, a configuration we refer to as FCAG. The jRCaMPla and jRGECO1a constructs included the nuclear export sequences found in the original publication (Dana et al., 2016). For nuclear localization, the nuclear export sequence of $\mathrm{jRGECO1a}$ was replaced with an $\mathrm{H} 2 \mathrm{~B}$ tag, and cloned into an AAV-hSyn-DO Cre-off vector. TsChR, including an $\mathrm{N}$-terminal Kir2.1 trafficking sequence followed by a GFP fluorescent tag, was cloned into FCK and into an AAV expression vector under control of the human synapsin promoter (AAV-hSyn). CheRiff-TS-GFP (Addgene plasmid 51693) was cloned into an AAV-CAG-DIO expression vector. FCK-ChR2(H134R)-GFP was used as a reference for eTsChR characterization. FCK-VSV-G (Addgene plasmid 8454) and psPAX2 (Addgene plasmid 12260) were used in lentiviral production. pUC19 (NEB N3041) was used as a diluent in calcium phosphate transfections.

\section{Cell culture and gene expression}

HEK cell culture and gene expression. Photophysical measurements of RGECIs were performed in HEK293T cells (ATCC, CRL-11268) cultured as previously described (Hochbaum et al., 2014). Cells were grown at $37^{\circ} \mathrm{C}, 5 \%$ $\mathrm{CO}_{2}$ in DMEM containing 10\% FBS (Life Technologies, 10082-147) and 50 $\mathrm{U} / \mathrm{ml}$ penicillin-streptomycin (Life Technologies, 15060-063). Cells were split with trypsin-EDTA (Life Technologies, 25300054) every 2-3 d and used before passage 25 . For gene delivery, cells were grown to $70 \%$ confluence in 24-well plates or $35 \mathrm{~mm}$ plastic dishes. Two hundred nanograms (for 24-well plates) or $400 \mathrm{ng}$ (for $35 \mathrm{~mm}$ plastic dishes) of FCAGRGECI DNA was transfected using TransIT-293 (Mirus, 2705) following manufacturer instructions. After $24 \mathrm{~h}$, cells were split onto Matrigel (Fisher Scientific, 356234) coated glass bottom plates (In Vitro Scientific, D35-14-1.5-N) and imaged $24 \mathrm{~h}$ later.

Low titer lentivirus production. HEK293T cells were cultured as in the previous section, except that cells were split daily and the cell density was always maintained between 30 and $70 \%$. Before P11, cells were split onto gelatin-coated plates, prepared by incubating $15 \mathrm{~cm}$ plastic dishes (Nunc) for $20 \mathrm{~min}$ at room temperature with $10 \mathrm{ml}$ EmbryoMax $0.1 \%$ Gelatin solution (Millipore, FS-006-B) and aspirating to dryness. Ten centimeter dishes were also used, and all amounts were scaled to the 
a

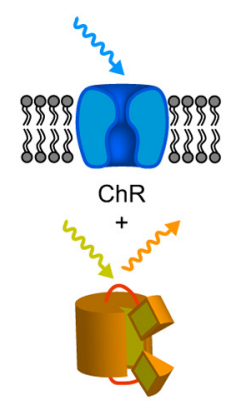

b
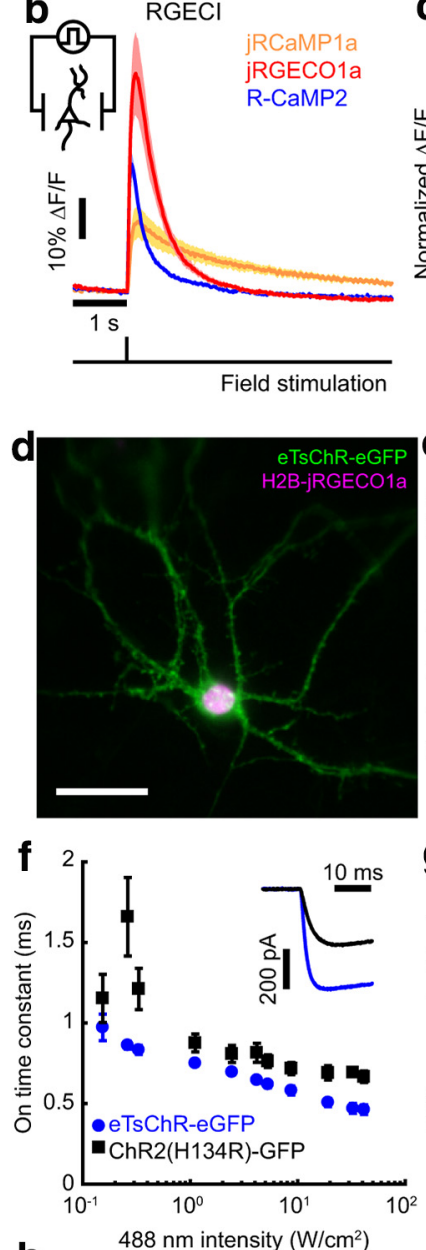

h

$488 \mathrm{~nm}$ intensity $\left(\mathrm{W} / \mathrm{cm}^{2}\right)$
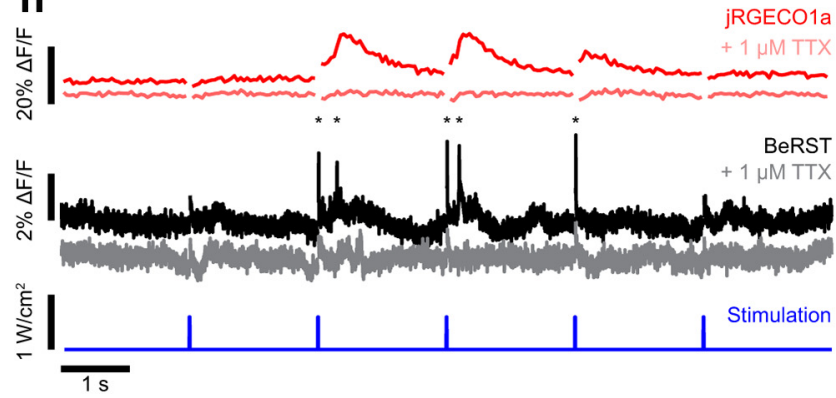

Figure 1. All-optical neurophysiology with a blue-shifted channelrhodopsin and a redshifted $\mathrm{Ca}^{2+}$ indicator. $\boldsymbol{a}$, Left, Schematic of a spectrally orthogonal channelrhodopsin and RGECI. Right, Action spectra of proteins used in this work. Spectra are reproduced with permission from Dana et al., 2016 for jRGEC01a; Klapoetke et al., 2014 for TsChR; and Hochbaum et al., 2014 for CheRiff. $\boldsymbol{b}$, Single action potential responses of RGECIs in cultured rat hippocampal neurons. Dark lines indicate the average of 3 FOVs, $\sim 30$ cells/FOV, for R-CaMP2, and 4 FOVs for jRGEC01 a and jRCAMP1a. Colored bands indicate \pm SEM. Dishes were stimulated with $1 \mathrm{~ms}$ field stimulation pulses. RGECl fluorescence was recorded at $50 \mathrm{~Hz}$.c, Kinetics of the RGECls, shown by plotting data in $\boldsymbol{b}$ normalized to peak $\Delta \mathrm{F} / \mathrm{F}$. $\boldsymbol{d}$, Cultured hippocampal neuron coexpressing smaller surface area. After cells reached $80 \%$ confluency, cells were switched to $16 \mathrm{ml}$ pre-warmed DMEM without FBS for $1-2 \mathrm{~h}$. For each dish, the following were added, in order, to $1.2 \mathrm{ml}$ DMEM: $14 \mu \mathrm{g}$ of FCK-RGECI plasmid, $9 \mu \mathrm{g}$ psPAX2, and $4 \mu \mathrm{g} \mathrm{VsVg}$ were combined with $36 \mu \mathrm{l}$ of $1 \mathrm{mg} / \mathrm{ml}$ PEI in water (Sigma-Aldrich, 408727). The tube was vortexed and incubated at room temperature for $10 \mathrm{~min}$. The mixture was then pipetted dropwise over the surface area of the dish and the cells were returned to the incubator for $4 \mathrm{~h}$. After the incubation, the medium was replaced with $16 \mathrm{ml}$ DMEM $+10 \%$ FBS without antibiotics. Thirtysix to $48 \mathrm{~h}$ later, the medium was collected and centrifuged for $5 \mathrm{~min}$ at $500 \times g$. The supernatant was filtered through a $0.45 \mu \mathrm{m}$ filter, blocked with DMEM $+10 \%$ FBS, and aliquoted in 1-5 ml fractions. Aliquots were kept at $-80^{\circ} \mathrm{C}$ until use.

Primary neuron culture and gene expression. Cultured rat hippocampal neurons on astrocyte monolayers were prepared as previously described (Hochbaum et al., 2014), with two modifications: (1) In Vitro Scientific dishes model D35-14-1.5-N were used instead of D35-20-1.5-N, while keeping the cell densities the same, and (2) neurons were cultured in Neurobasal-A (Life Technologies, 10888-022) supplemented with B27 (Life Technologies, 17504044) instead of BrainBits' NbActiv4. For electrophysiological and AON measurements, neurons were transfected via calcium phosphate, as previously described (Hochbaum et al., 2014) at 7 DIV and used at 14-16 DIV. For comparison of RGECI performance by field stimulation (Fig. 1b,c), cultured neurons were lentivirally transduced. At 7 DIV, half of the media from each dish $(1 \mathrm{ml})$ was reserved and replaced with $250 \mu \mathrm{l}$ of low-titer FCK-RGECI lentivirus. After $2 \mathrm{~d}$, all of the media was removed and replaced with the reserved media supplemented with an additional $1 \mathrm{ml}$ of Neurobasal-A + B27 supplement.

\section{Imaging and electrophysiology in culture}

Microscope. A custom-built epifluorescence microscope was used for measurements in HEK293T cells and in cultured neurons. Illumination was provided by a $561 \mathrm{~nm} 100 \mathrm{~mW}$ laser (Cobolt Jive 0561-04-01-0100$500)$ or a $488 \mathrm{~nm} 100 \mathrm{~mW}$ laser (Coherent Obis 1226419). The laser lines were combined and focused on the back focal plane of the objective (Olympus Fluor $4 \times 0.24$ NA for single action potential measurements of RGECIs; Olympus LCPlanFL $20 \times 0.40$ NA for RGECI photobleaching measurements; Olympus UPlanSApo $10 \times 0.40$ NA for RGECI photoswitching characterization; Olympus ApoN $60 \times 1.49$ NA Oil for eTsChR characterization). Fast modulation of the $488 \mathrm{~nm}$ laser was achieved with an acousto-optic tunable filter (Gooch \& Housego, TF525-250-6-4GH18A). Both laser lines were additionally modulated by neutral density filters as necessary. Fluorescence light was separated from illumination light using a quadband dichroic (Semrock Di01-R405/488/561/635). HQ550/50m or ET595/50 bandpass emission filters (Chroma) were used to isolate GFP or RGECI fluorescence, respectively, before capturing on a scientific CMOS camera (Hamamatsu, Orca Flash 4.0). For photobleaching measurements, an additional 1 OD filter was inserted in the imaging path to avoid saturating the camera. Illumination profiles were acquired on bead samples before experiments each day and spot size was determined using a $1 / \mathrm{e}^{2}$ cutoff. Laser powers were measured at the sample plane. A digital acquisition (DAQ) card (National Instruments, PCIe

H2B-jRGEC01a (magenta) and eTsChR (green). Scale bar, $10 \mu \mathrm{m} . \boldsymbol{e}$, Steady-state photocurrents of eTsChR and ChR2 (H134R) in cultured neurons held at $-65 \mathrm{mV}$ (1 s pulses, $488 \mathrm{~nm}, n=6$ cells for each construct). Inset, Photocurrent response to $2 \mathrm{~W} / \mathrm{cm}^{2} 488 \mathrm{~nm}$ illumination. $f$, Channelrhodopsin activation time constant as a function of $488 \mathrm{~nm}$ illumination intensity. Inset, Photocurrents during illumination start. $\boldsymbol{g}$, Closing time constants. Inset, Photocurrents during illumination stop. $\boldsymbol{h}$, Optogenetic stimulation induced action potentials and corresponding fluorescence transients in a cultured neuron expressing jRGECO1a and eTsChR. Pulses of blue light $\left(488 \mathrm{~nm}, 10 \mathrm{~ms}, 680 \mathrm{~mW} / \mathrm{cm}^{2}\right)$ drove action potentials $\left({ }^{*}\right)$, which were identified via fluorescence of a far-red voltage-sensitive dye, BeRST1 (1 $\mu$ m, black; Huang et al., 2015). Fluorescence transients of jRGEC 01 a accompanied action potentials (red). TTX $(1 \mu \mathrm{M})$ silenced activity in both the voltage (pink) and $\mathrm{Ca}^{2+}$ (gray) channels, confirming that signals arose from neural activity and not optical crosstalk. Voltage imaging was performed at $500 \mathrm{~Hz}$ with $0.7 \mathrm{~W} / \mathrm{cm}^{2} 640 \mathrm{~nm}$ light and calcium imaging was performed at $20 \mathrm{~Hz}$ with $1.1 \mathrm{~W} / \mathrm{cm}^{2} 561 \mathrm{~nm}$ light. All error bars indicate mean \pm SEM. 
Table 1. In vitro characterization of RGECls

\begin{tabular}{lllll}
\hline Construct & Single AP $\max \Delta \mathrm{F} / \mathrm{F}, \%$ & $\tau_{\text {on, ms }}$ & $\tau_{\text {off, } \mathrm{ms}}$ & $\tau_{\text {bleach, }}$ \\
\hline jRGEC01a & $54 \pm 10, n=4 \mathrm{FOV}, \sim 30$ cells/FOV & $47.2 \pm 1.0$ & $443 \pm 38$ & $80.5 \pm 5.1, n=9$ cells \\
R-CaMP2 & $31 \pm 3, n=3 \mathrm{FOV}, \sim 30$ cells/FOV & $26.3 \pm 1.0$ & $271 \pm 20$ & $61.9 \pm 2.8, n=8$ cells \\
jRCaMP1a & $17 \pm 4, n=4$ FOV, $\sim 30$ cells/FOV & $61.2 \pm 2.1$ & $1600 \pm 160$ & $37.8 \pm 2.1, n=8$ cells \\
\hline
\end{tabular}

Quantification of action potential responses in cultured neurons in Figure 1, and photobleaching kinetics in HEK293T cells. Action potential magnitudes and sensor kinetics are from 3 FOVs for R-CaMP2 and 4 FOVs for jRGEC01a and jRCaMP1a in separate dishes. Dishes were stimulated with $1 \mathrm{~ms}$ field stimulation pulses while imaging RGECI fluorescence at $50 \mathrm{~Hz}$ with $2.45 \mathrm{~W} / \mathrm{cm}^{2} 561 \mathrm{~nm}$ illumination. Photobleaching measurements were performed in HEK293T cells under 44 $\mathrm{W} / \mathrm{cm}^{2} 561 \mathrm{~nm}$ illumination (compared with $0.1 \mathrm{~W} / \mathrm{cm}^{2}$ used in slice imaging). All values are reported as mean $\pm \mathrm{SEM}$.
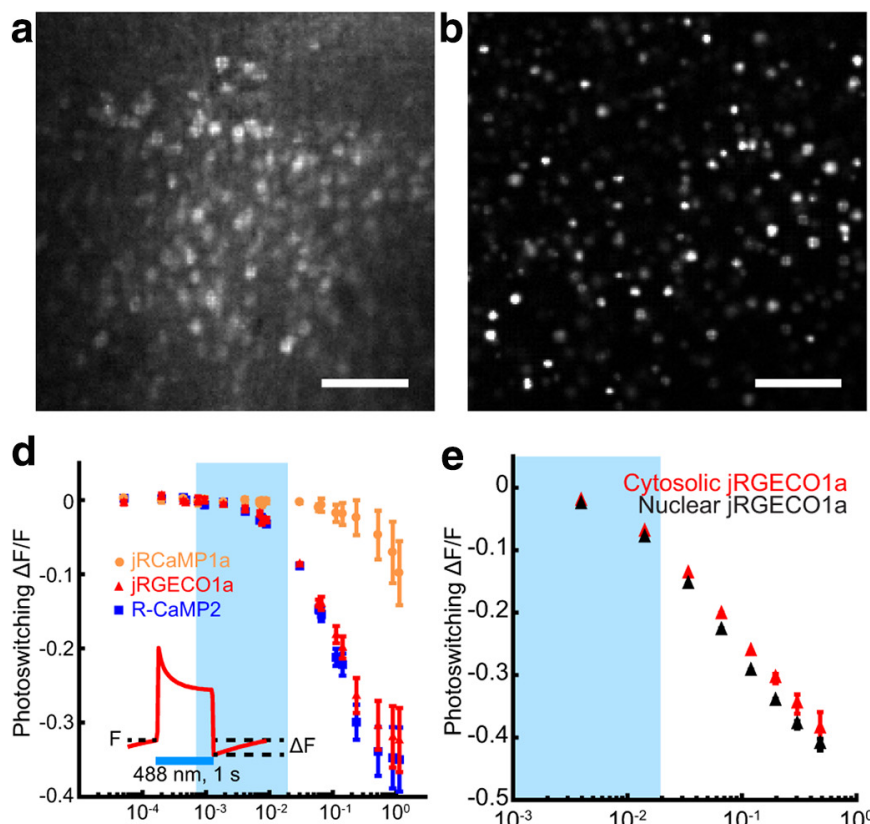

$488 \mathrm{~nm}$ illumination dose $\left(\mathrm{J} / \mathrm{cm}^{2}\right.$ in $\left.1 \mathrm{~s}\right)$

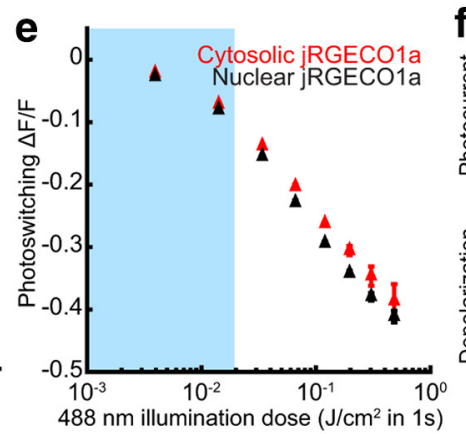

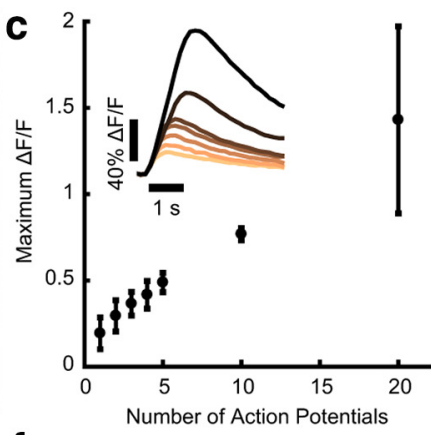

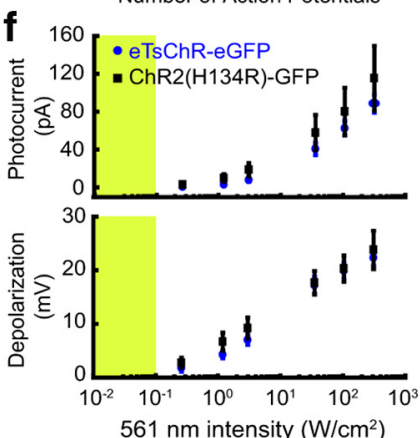

Figure 2. Characterization of soma-localized RGECls and eTsChR. $\boldsymbol{a}, \boldsymbol{b}$, Maximum intensity projections of Hadamard $z$-stacks from acute cortical slices prepared from mice injected with (a) cytosolic AAV1-syn-NES-jRGEC01a or (b) nuclear-targeted AAV9-syn-D0-H2B-jRGEC01a. Scale bars, $100 \mu \mathrm{m}$. c, Maximum $\Delta \mathrm{F} / \mathrm{F}$ of H2B-jRGEC01a fluorescence versus number of evoked action potentials in cultured neurons, stimulated via current injection ( $n=3$ cells). Inset: example responses to increasing numbers of action potentials. $\boldsymbol{d}$, Blue light induced photoswitching of cytosolic RGECIs in HEK293T cells under basal $\mathrm{Ca}^{2+}$ levels. RGECI fluorescence was recorded at $50 \mathrm{~Hz}$ with illumination at $561 \mathrm{~nm}, 80 \mathrm{~mW} / \mathrm{cm}^{2}$. Blue illumination ( $1 \mathrm{~s} \mathrm{pulses,} 488 \mathrm{~nm}$ ) was added to the yellow illumination. Photoswitching was quantified as the decrease in fluorescence following blue light illumination compared with the initial fluorescence (inset); $n=3 \mathrm{FOV}, \sim 50 \mathrm{cells} / \mathrm{FOV}$, for each construct. e, Comparison of photoswitching in cytosolic and nuclear-localized jRGECO1a. Blue bar represents range of illumination doses used for optogenetic stimulation in this study. $f$, Activation of channelrhodopsins as a function of $561 \mathrm{~nm}$ illumination intensity. Top, Steady-state photocurrents in cultured neurons voltage-clamped at $-65 \mathrm{mV}$. Bottom, Voltage depolarization under current-clamp with an initial potential of $-65 \mathrm{mV}$. Yellow bar indicates typical jRGECO1a imaging intensities. Acquired from cultured rat hippocampal neurons; $n=6$ for each construct. All error bars indicate mean \pm SEM.

6259) was used to synchronize command and recording waveforms. Imaging frame rates and illumination powers are indicated in figure captions for each experiment.

Imaging and electrical recordings. In all imaging measurements, culture medium was replaced with imaging buffer containing the following (in mu): $125 \mathrm{NaCl}, 2.5 \mathrm{KCl}, 2.5 \mathrm{HEPES}, 30$ glucose, $1 \mathrm{MgCl}_{2}, 3 \mathrm{CaCl}_{2}$. The buffer $\mathrm{pH}$ was adjusted to 7.3 and osmolarity was $310 \mathrm{mOsm}$. Measurements were performed at room temperature. Ten micromolar CNQX, 20 $\mu \mathrm{M}$ gabazine, and $25 \mu \mathrm{M}$ APV (all Tocris Bioscience) were included in cultured neuron experiments to block synaptic transmission. Channelrhodopsin characterization measurements were performed in synaptic blockers with the addition of $1 \mu \mathrm{M}$ tetrodotoxin (Tocris Bioscience). No additional all-trans retinal was added.

Field stimulation (Fig. 1b,c) was performed by inserting two chlorided silver wire loops $2 \mathrm{~cm}$ apart into the glass-bottomed imaging dish, touching the plastic on either side of the coverslip. A high voltage amplifier (Krohn-hite $7600 \mathrm{M}$ ) was used to amplify $1 \mathrm{~ms}$ pulses generated by the DAQ card to $60-120 \mathrm{~V}$. Three to 4 FOVs were acquired for each construct, using a fresh dish each time.

For patch-clamp electrophysiology measurements (Figs. 1e-g, 9d), 3-5 M $\Omega$ borosilicate glass pipettes (WPI) were filled with internal solution containing the following (in $\mathrm{mM}$ ) 125 potassium gluconate, $8 \mathrm{NaCl}$, $0.6 \mathrm{MgCl}_{2}, 0.1 \mathrm{CaCl}_{2}, 1$ EGTA, 10 HEPES, $4 \mathrm{Mg}$-ATP, $0.4 \mathrm{Na}$-GTP, adjusted to $\mathrm{pH} 7.5$ and $295 \mathrm{mOsm}$ with sucrose. Voltage- and currentclamp recordings were obtained with a MultiClamp 700B amplifier (Molecular Devices) while illuminating with $1 \mathrm{~s} 488 \mathrm{~nm}$ pulses or $2 \mathrm{~s} 561 \mathrm{~nm}$ pulses of intensities indicated in the figure captions. In voltage-clamp measurements, cells were held at $-65 \mathrm{mV}$. In current-clamp measurements, an offset current was injected to maintain the resting membrane potential at $-65 \mathrm{mV}$. Signals were filtered at $5 \mathrm{kHz}$ with the amplifier's internal Bessel filter and digitized at $10 \mathrm{kHz}$.

Data analysis. All values are expressed as mean \pm SEM. $P$ values were obtained from Student's $t$ tests unless otherwise indicated.

Whole FOV RGECI single action potential responses (Fig. 1b,c; Table 1) were extracted as previously described (Chen et al., 2013). Activation time constants were extracted from monoexponential fits between stimulation onset and maximum $\Delta \mathrm{F} / \mathrm{F}$. For inactivation time constants, the fluorescence trace after the maximum $\Delta \mathrm{F} / \mathrm{F}$ was fit to a sum of two exponential decays, and the $\tau_{\text {off }}$ was taken as the time for the fit to decay to half its maximum value. Photobleaching traces (Table 1) were extracted from separate cells and fit to a monoexponential to obtain time constant $\tau_{\text {bleach }}$.

Movies of blue-light photoswitching (Fig. $2 d, e$ ) were preprocessed to reject saturated pixels and a threshold equal to half the average of movie was used to separate foreground from background. Background intensity was subtracted from the original movies and the averages of the resulting 
a

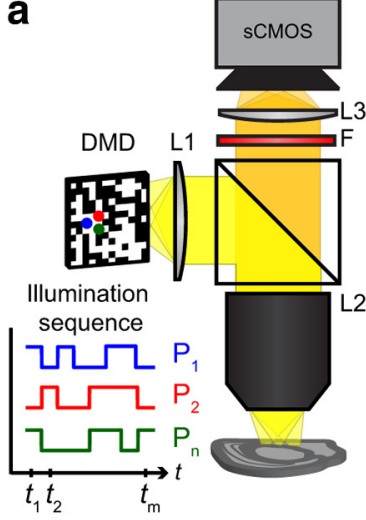

C

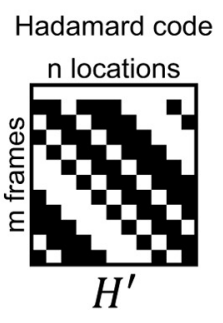

b Camera

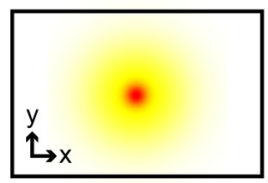

Sample

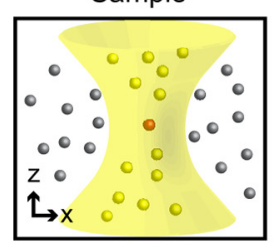

1. Acquire

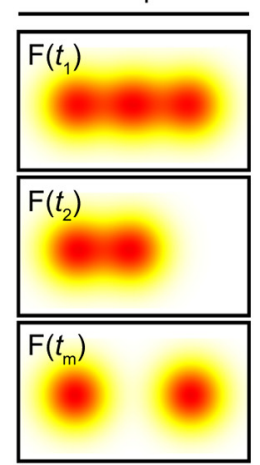

d

$$
\text { c }
$$

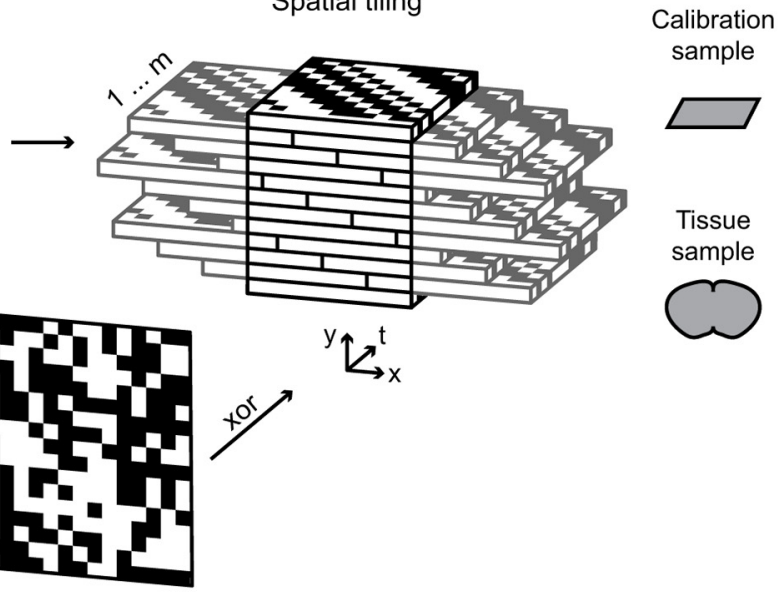

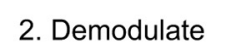

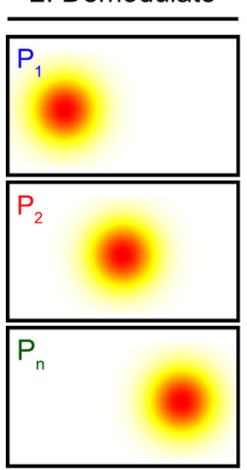

Raw images
3. Apply aperture

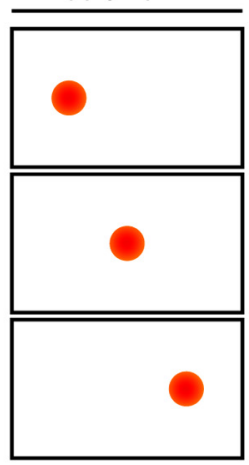

Correlations with Hadamard patterns
4. Reconstruct

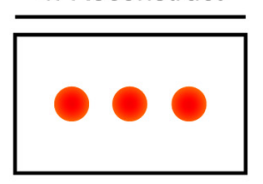

Product: confocal pinholes
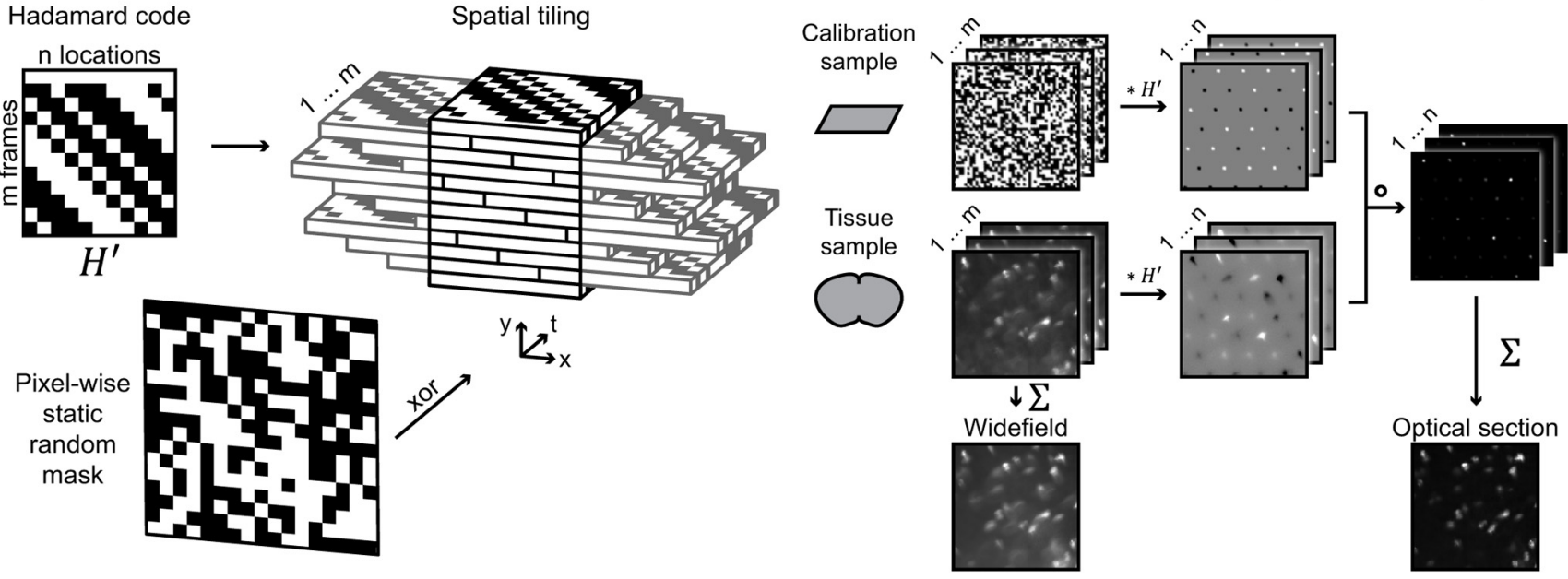

Figure 3. Optical sectioning by Hadamard microscopy. $\boldsymbol{a}$, Schematic of ultra-wide-field microscope, showing orthogonal illumination sequences in neighboring DMD pixels $\left(P_{1}, P_{2}, \ldots, P_{n}\right)$. The full sequences have a $50 \%$ duty cycle. $\boldsymbol{b}$, Left, In a thick, scattering sample, the in-focus light (red) is dispersed by scattering and mixed with out-of-focus light (yellow). Right, Hadamard microscopy protocol. (1) The sample is illuminated with orthogonal functions of time at adjacent points in space. (2) The images are demodulated by matched filtering with the illumination sequence. (3) Scattered light is rejected by a software aperture. (4) The optically sectioned image is reconstructed from a sum of the demodulated images. c, Codes from a Hadamard matrix were tiled to fill image space. The number of elements in the Hadamard code determined the number of frames in the pattern sequence. A random mask was applied to invert the code in $50 \%$ of illumination pixels, yielding pseudorandom patterns with flat spatial and temporal power spectra. $\boldsymbol{d}$, Raw images were acquired in a calibration sample (a thin homogeneous fluorescent film) and a tissue sample, one frame per Hadamard pattern. Cross-correlation maps between microscope data and Hadamard codes produced arrays of peaks corresponding to signals from distinct sample regions. Negative peaks corresponded to pixels whose Hadamard sequence was inverted. Pixel-wise multiplication of the demodulated images from the calibration sample and from the tissue sample led to multipoint confocal images. These images were summed to produce an image reconstruction. Asterisk $\left(^{*}\right)$ indicates matrix multiplication. Detailed description in Materials and Methods.

foreground traces (combining 10-20 cells each) were used in downstream analysis. Traces were converted to $\Delta \mathrm{F} / \mathrm{F}$ using the fluorescence value before blue light stimulation as $\mathrm{F}_{0}$. "Photoswitching $\Delta \mathrm{F} / \mathrm{F}$ " was defined as the $\Delta \mathrm{F} / \mathrm{F}$ immediately after blue-light illumination ends (Fig. $2 d$, inset).

For comparison of channelrhodopsins (Figs. $1 e-g, 9 d$ ), cells were rejected if they required $>100 \mathrm{pA}$ holding current to maintain $-65 \mathrm{mV}$ in current-clamp or if their baselines drifted by more than the smallest steady-state photocurrent amplitude in voltage-clamp mode. Steadystate $488 \mathrm{~nm}$ photocurrents were extracted as the average photocurrent over the last $100 \mathrm{~ms}$ of blue-light illumination. Steady-state $561 \mathrm{~nm}$ photocurrents and depolarizations were extracted from $1 \mathrm{~s}$ of data. On time constants were obtained from single exponential fits to the first 1.5 $\mathrm{ms}$ of $488 \mathrm{~nm}$ illumination. Off time constants were obtained from single exponential fits to the $99.5 \mathrm{~ms}$ following blue-light 488 illumination.

Recordings of jRGECO1a fluorescence in Figure $1 \mathrm{~h}$ were corrected for photobleaching with a bi-exponential fit to the initial period in each movie, before stimulation, while recordings of BeRST1 fluorescence were corrected for photobleaching by a sliding, 1000 point, median filter. Both traces were converted to $\Delta \mathrm{F} / \mathrm{F}$ based on the fluorescence before blue-light stimulation. Frames acquired during blue light stimulation were dropped to avoid optical crosstalk.

\section{Hadamard imaging}

Microscope. In the ultra-wide-field microscope (Fig. 3a), a $561 \mathrm{~nm}$ laser beam (MPB Communications, F-04306-02) was transmitted through a rotating diffuser, and merged with a $470 \mathrm{~nm}$ LED beam (Thorlabs, M470L3). Both were expanded, focused, and coupled through free space to fill with high NA illumination a digital micromirror device (DMD) module (Vialux V-7001; $1024 \times 768$ pixels, $13.7 \mu \mathrm{m}$ pitch). Multiple diffraction orders emitted from the DMD pattern were transmitted by a $100 \mathrm{~mm}$ projection tube lens (Zeiss, Makro-Planar $100 \mathrm{~mm}$; Fig. 3a, L1), reflected off a custom dichroic mirror (Semrock Di01-R405/488/561/ 635-t3-60x85), and imaged onto the sample by a $50 \mathrm{~mm}$ objective lens (Olympus, MVPLAPO 2XC, NA 0.5; Fig. 3a, L2). The $3 \mathrm{~mm}$ substrate thickness of the dichroic mirror minimized warping-induced projection aberrations. Fluorescence emission was collected through the same objective and dichroic, a large diameter $(60 \mathrm{~mm})$ emission filter (Semrock FF01-520/35-60-D or Chroma ET600/50m; Fig. 3a, F), and a $135 \mathrm{~mm}$ imaging tube lens (Zeiss, Apo-Sonnar 135 mm; Fig. 3a, L3) onto a scientific CMOS camera (Hamamatsu, Orca Flash 4.0, $2048 \times 2048$ pixels). The FOV was $4.6 \times 4.6 \mathrm{~mm}^{2}$ in the sample plane, corresponding to a magnification of $2.89 \times$ onto the camera, and $2.17 \times$ onto the DMD. Camera and DMD pixels were 2.25 and $6.3 \mu \mathrm{m}$ wide in the sample, 
respectively. Hardware and triggers were programmed in LabVIEW, with pattern generation and data analysis performed in MATLAB.

Illumination patterns. To reject light scattered within the sample, pattern sequences were designed such that in the projected series of $2 \mathrm{D}$ images, neighboring locations of the sample were illuminated with orthogonal functions of intensity versus time. A Hadamard matrix, $H$, of size $m$ is a binary square matrix with elements $\{-1,1\}$ that fulfills $H^{T} H=m I_{m}$, where $I_{m}$ is the identity matrix of size $m$; its normalized form has value 1 in the first column and first row. Illumination intensities could not be negative, so the projected intensity patterns were defined as $P=\left(H^{\prime}+1\right) / 2$, where $H^{\prime}=H[1, \ldots, m ; m-n+1, \ldots, m]$ was an incomplete orthogonal basis given by the last $n$ columns of a normalized Hadamard matrix, with $n<m$.

The illumination patterns $P$ thus had binary values $\{0,1\}$ corresponding to DMD mirror positions OFF and ON respectively. Each location was illuminated with a positive temporal function orthogonal to all other designed Hadamard codes, as verified by $P^{T} H^{\prime}=I_{n} * m / 2$. For a given number of locations, a Hadamard matrix provided a set of shortest possible binary orthogonal functions. To arrange the $n$ codes in $P$ into illumination patterns, $m=n+1$ images were defined assigning code $k_{i, j} \in\{1 . . n\}$ to DMD pixel $(i, j)$, as $k_{i, j}=\bmod (i * q+j, n)+1$, where $q$ was an offset parameter that maximized spatial separation of repeated codes. $(n, q)$ was set to $(11,3)$ for functional imaging, and to $(63,14)$ or $(59,8)$ for structural imaging. To further reduce spurious scattering cross talk, a random binary mask $R$ was generated to flip the sign of $50 \%$ of DMD pixels, applied as an exclusive OR operation on all DMD patterns against the same mask, $R$. In the compressed sensing experiment (see Fig. 10) the sequence of Hadamard patterns was interleaved with its complement (interchanging bright and dark pixels).

Calibration. To prepare the system for each imaging session, a calibration data-set $C$ was obtained by placing a thin fluorescent sample at the focal plane, and acquiring an image with each illumination pattern. The sample consisted of green or orange neon Sharpie (Newell Brands) ink painted on (or sandwiched between) glass coverslips, to match imaging conditions of subsequent acute (fixed) tissue experiments. For each camera pixel, the time series of its photon counts was cross-correlated against each Hadamard sequence as $C^{T} H^{\prime}$. The resulting cross-correlation images displayed sharp peaks indicating the projected DMD locations for each code, with positive or negative correlation given by $R$. A synthetic approximation to the cross-correlation maps was calculated by finding the code with maximum absolute correlation for each pixel, yielding homogeneous, noise-free cross-correlation maps.

Reconstruction. A Hadamard sequence data-set $D$ was acquired after replacing the calibration sample with a tissue sample. Photon counts at each camera pixel were cross-correlated against each Hadamard sequence as $D^{T} H^{\prime}$. Cross-correlation images displayed a set of peaks modulated by the local fluorophore density, and broadened by off-focus fluorescence and light scattering in the sample. Each peak characterized the scattering function of the corresponding tissue location, i.e., its absolute value represents the image one would record with an illumination spot focused solely at that location in the tissue. The next step was to apply a set of computational "pinholes" to select the unscattered in-focus photons, and to reject all others. The spatial filter was implemented through the element-wise product of calibration correlation maps and tissue correlation maps, resulting in the positive filtered maps $F=C^{T} H^{\prime} \circ D^{T} H^{\prime}$, where $\circ$ represents element-wise multiplication. This computational process was akin to sifting emitted light through an array of pinholes as happens physically in spinning disk confocal microscopy. The final computation step was to aggregate the unscattered light by direct sum of the filtered images over all code maps, defining an optical section image $\phi_{i}=\sum_{k=1}^{n} F_{i, k}$.

To correct for slight motion artifacts because of sample drift, all datasets from one brain slice were registered to a reference image using a $b$-splines transform maximizing mutual information (Klein et al., 2010).

Compressed Hadamard imaging. While this manuscript was in review, we developed a compressed sensing measurement method to increase the time resolution from $m$ camera frames (where $m$ is the length of the Hadamard sequence) to 2 camera frames per optical section. This approach and its tradeoffs were characterized previously (Parot et al., 2019). For application of compressed Hadamard imaging to AON (see
Fig. 10), procedures, optogenetic methods, and imaging protocol were as in other experiments, except an Olympus XLPLN10XSVMP $(\mathrm{NA}=0.6)$ objective was used to map DMD and camera pixel size to 2.52 and $0.9 \mu \mathrm{m}$ at the sample respectively. Illumination comprised 24 patterns, interleaved with their complements, repeated in 36 cycles. Camera and DMD frames were updated at $66 \mathrm{~Hz}$. Reconstruction was performed in $64 \times 64$ pixel blocks with 40 principal components per block.

All static Hadamard image computations in this work were accelerated by computing $\phi_{i}=\sum_{k=1}^{m} G_{i, k}$, with $G=C^{T} \circ D^{T}$. Both approaches are numerically equivalent, as proved by:

$$
\begin{aligned}
\phi_{i} & =\sum_{k=1}^{n} F_{i, k}, \\
& =\sum_{k=1}^{n} \sum_{p=1}^{m} C_{i, p} H_{k, p} \sum_{q=1}^{m} D_{i, q} H_{k, q}, \\
& =\sum_{p=1}^{m} \sum_{q=1}^{m} C_{i, p} D_{i, q} \sum_{k=1}^{n} H_{k, p} H_{k, q}, \\
& =\sum_{p=1}^{m} \sum_{q=1}^{m} C_{i, p} D_{i, q} \delta_{p, q}, \\
& =\sum_{q=1}^{m} C_{i, q} D_{i, q}, \\
& =\sum_{k=1}^{m} G_{i, k},
\end{aligned}
$$

where $\delta_{p, q}$ is a Kronecker delta. The resulting optical section preserved unscattered light emitted from the focal plane, while rejecting scattered light and background emissions. Standard wide-field epifluorescence images were also computed from each Hadamard dataset by computing a direct sum of all frames in the raw images, $W_{i}=\sum_{k=1}^{m} D_{i, k}$.

Hadamard image formation. To understand the optical sectioning process, Hadamard microscopy was modeled as an incoherent illumination, intensity-linear space-invariant optical system in which the intensity after propagation is given by a convolution between intensity before propagation and an intensity impulse response function. In a discrete representation, the circulant convolution matrix $S_{1}^{T}$ represented threedimensional excitation intensity at the object, in response to an impulse function reflectance at the DMD plane (turning on one DMD pixel). Similarly, $S_{2}$ was defined as the intensity collected by an impulse detector at the camera plane from emitted fluorescence in a three-dimensional object (analogous to detection from 1 camera pixel). The data collected from tissue with fluorophore distribution $G$ upon illumination with a structured illumination pattern $P$ was represented as $D=S_{2} \operatorname{diag} G S_{1}^{T} P$, where diag denoted rearrangement between vector and diagonal matrix. Calibration with a thin uniform fluorescent object and no scattering was represented as $C=P$. After assuming that $P$ contains an orthonormal Hadamard code with no spatial repetition, it followed that $C^{T} H^{\prime}=I_{n}$ and $D^{T} H^{\prime}=S_{2} \operatorname{diag} G S_{1}^{T} I_{n}$. Then $\phi=\Sigma_{k} C^{T} H^{\prime} \circ D^{T} H^{\prime}=\operatorname{diag}\left(S_{2} \operatorname{diag} G S_{1}^{T}\right)$, or $\phi=\left(S_{1} \circ S_{2}\right) G$. The reconstructed optical section $\phi$ was proportional to the object $G$ convolved with the confocal scattering function $S=$ $S_{1} \circ S_{2}$, which resulted from the element-wise product of the projection and collection scattering functions. To simulate the effects of lateral subpixel offset between the DMD pixels and the smaller camera pixels, we used a continuous space version of the model, defining the diffractionlimited excitation and emission point-spread functions (PSFs) as Gaussian functions and convolving these PSFs by square apertures representing the DMD and camera pixels, respectively. The system PSF was calculated from the product of the excitation and emission PSFs.

Image processing and filtering. The lines between DMD pixels led to a periodic grid artifact in Hadamard optical sections. A Gaussian stopband 
filter was used to attenuate these artifacts. The filter parameters were not changed after initial setup.

The size of the computational pinholes could be adjusted in software to trade optical signal level for $z$-resolution. Tuning of pinhole sizes was achieved by applying a spatial Gaussian filter to the calibration patterns, with $\sigma=5.6 \mu \mathrm{m}$ for functional images, and $\sigma=3.4 \mu \mathrm{m}$ for structural images. Further increases in $\sigma$ to sizes larger than the spacing of pinholes resulted in a continuous transition to wide-field epifluorescence imaging.

An additional source of systematic error came from local inhomogeneity of illumination patterns. Although the projected patterns have $50 \%$ duty cycle on average, variations in local illumination can change the relative contributions of in-plane signal and background, resulting in imperfect background cancellation manifested as regions with periodic background artifacts. This effect was minimized for Hadamard images in Figure 5, $b$ and $c$, by dividing raw tissue data by its low spatial frequency component, calculated with a Gaussian filter with $\sigma=22.5 \mu \mathrm{m}$. Images in all figures were linearly mapped to grayscale setting 0 to black and saturating to white the 0.01 percentile of highest intensity values unless otherwise indicated.

Characterization. We quantified the performance of Hadamard, stripe SIM, and HiLo optical sectioning methods by three measurements. First, we measured the PSF by imaging $200 \mathrm{~nm}$ fluorescent beads (Invitrogen, F8763) embedded in 1.5\% agarose gel. Second, we tested the in-plane uniformity of optical sections by measuring a thin fluorescent plane of orange neon Sharpie (Newell Brands) ink painted on a glass coverslip. Third, we acquired multiplane images of an acute brain slice expressing $\mathrm{H} 2 \mathrm{~B}-\mathrm{jRGECO} 1 \mathrm{a}$ to evaluate the imaging quality of each method in turbid tissue.

For the beads and plane experiments, illumination patterns for Hadamard codes of length 12, together with striped illumination with period 4 pixels and four phases, were interleaved and repeat-averaged to match total photons and photobleaching conditions across datasets. HiLo optical sections were computed from the same patterns used for Hadamard imaging, using a photon-matched uniform illumination image and a repeat-averaged structured image corresponding to one Hadamard pattern. HiLo uses only a single random illumination pattern, and thus necessarily uses a non-uniform total photon count across the sample. We used more total photons in HiLo optical sections to avoid penalizing this method in the comparison. A series of images taken at $\Delta z=2.24 \mu \mathrm{m}$ were acquired to map the three-dimensional PSF.

Hadamard images were calculated as $\phi_{i}=\sum_{k=1}^{m} G_{i, k}$, with $G=C^{T} \circ D^{T}$. Stripe SIM optical sections were calculated as $\phi=\left|\sum_{k=0}^{m-1} I_{k} e^{-i 2 \pi k / m}\right|$, with $m=$ 4. HiLo optical sections were calculated setting the wavelet filter $\sigma=0.75$. DMD modulation grid artifacts were present in all datasets and were not corrected. Wide-field reference images were obtained by summing all patterns in the Hadamard sequence.

Images of the homogeneous fluorescent plane were acquired following the same protocol as for the beads. The same flat field correction was applied to all datasets by subtracting the offset and dividing by the blurred intensity distribution of a focused wide-field image. All datasets were filtered equally to reduce DMD grid artifacts. Within a region-ofinterest, the SD of values was normalized by their mean to obtain coefficients of variation.

To estimate the degree of cell-to-cell fluorescence crosstalk in nuclearlabeled acute brain slices, we first computed a mean Hadamard "nucleus spread function", i.e., the mean fluorescence distribution measured from multiplane structural Hadamard images of fluorescent nuclei in acute brain slices. We then used high-resolution confocal microscopy to estimate the center locations of all neuronal nuclei in a fixed brain slice up to a depth of $100 \mu \mathrm{m}$. We computationally positioned the nucleus spread functions at the nuclear locations, adjusted signal levels to account for the measured attenuation with depth, and estimated the crosstalk, i.e., the amount of signal ascribed to each nucleus that originated from other nuclei.

\section{Software accessibility}

The Hadamard control and analysis software and an example data-set are available at: https://github.com/adamcohenlab/Hadamard-Code.

The compressed Hadamard analysis software is available at: https:// github.com/adamcohenlab/Compressed-Hadamard-Code.

\section{Animals and acute slice measurements}

Animals. All procedures involving animals were in accordance with the National Institutes of Health Guide for the care and use of laboratory animals and were approved by the Institutional Animal Care and Use Committee at Harvard University. Excitability measurements and characterization of functional Hadamard imaging were performed in wild-type C57BL6 (Charles River Laboratoires, 027) mice. Functional connectivity assays were performed in $\mathrm{Rbp} 4-\mathrm{Cre}^{+/-}$mice donated by Bernardo Sabatini's lab and originally generated in the GenSat project (KL100). For structural imaging of membrane bound mCitrine, FLOXed Optopatch-3 mice (Jackson Laboratories, 029679) were crossed with $\mathrm{Rbp}_{4}-\mathrm{Cre}^{+/-}$mice or with CaMK2a-Cre ${ }^{+/-}$mice (Jackson Laboratories, 005359).

AAV injection. AAV2/9-hSyn-DO-H2B-jRGECO1a $\left(1.60 \times 10^{13} \mathrm{GC} /\right.$ $\mathrm{ml})$ and AAV2/9-hSyn-eTsChR $\left(2.22 \times 10^{13} \mathrm{GC} / \mathrm{ml}\right)$ were produced at the Massachusetts Eye and Ear Infirmary Vector Core. AAV2/9-CAGDIO-CheRiff-TS-GFP $\left(5.80 \times 10^{13} \mathrm{GC} / \mathrm{ml}\right)$ was produced by the Stanford Vector Core. AAV1-hSyn-NES-jRGECO1a $\left(2.44 \times 10^{13} \mathrm{GC} / \mathrm{ml}\right)$ was purchased from the University of Pennsylvania Vector Core. When two viruses were coinjected, they were mixed in a 1:1 volume ratio. The final mixture was mixed in a 7:1 ratio with $0.4 \%$ Trypan blue to aid in visualization during injection. For viral injections, neonatal (P0-P2) animals were cold-anesthetized and taped to an aluminum heatsink submerged in an ice bath, with their heads resting on a modeling clay support. A stereotaxic injector (WPI, UMC4) mounted on a stereotaxic frame (Stoelting) was used to inject virus $1.6 \mathrm{~mm}$ anterior and $1.6 \mathrm{~mm}$ lateral to lambda every $0.4 \mathrm{~mm}$ starting from $3 \mathrm{~mm}$ beneath the surface of the skull. Forty nanoliters of virus was delivered at each depth at a rate of $5 \mathrm{nl} / \mathrm{s}$. If only one virus was used, only $20 \mathrm{nl}$ were injected per depth. Expression levels were sufficiently high for Hadamard imaging from $12 \mathrm{~d}$ until at least 9 weeks after injection.

Preparation of fixed slices. Fresh $300 \mu \mathrm{m}$ brain sections were incubated in $4 \%$ paraformaldehyde overnight at $4^{\circ} \mathrm{C}$, then mounted on a glass slide in Fluoromount and stored at $4^{\circ} \mathrm{C}$.

Acute slice preparation and imaging. Acute slices were prepared from P21-P28 animals. Animals were deeply anesthetized via isoflurane inhalation and transcardially perfused with ice-cold choline cutting solution, containing, in $\mathrm{mm} 110$ choline chloride, 25 sodium bicarbonate, 2.5 potassium chloride, 7 magnesium chloride, 0.5 calcium chloride, 1.25 monobasic sodium phosphate, 25 glucose, 11.6 ascorbic acid, and 3.1 pyruvic acid $(310 \mathrm{mOsm} / \mathrm{kg})$. The brain was blocked with one coronal cut just anterior to the tectum and mounted with Krazy glue on the specimen disk of a Leica VT1200s vibratome. After mounting, hemispheres were separated with a sagittal cut down the midline of the brain. The brain was covered with more ice-cold choline solution and then sliced in $300 \mu \mathrm{m}$ steps. Slices containing the striatum were recovered for $45 \mathrm{~min}$ in a $34^{\circ} \mathrm{C}$ artificial-CSF (ACSF) bath containing the following (in mм) $125 \mathrm{NaCl}, 2.5 \mathrm{KCl}, 25 \mathrm{NaHCO}_{3}, 2 \mathrm{CaCl}_{2}, 1 \mathrm{MgCl}_{2}, 1.25 \mathrm{NaH}_{2} \mathrm{PO}_{4}$, 25 glucose $(295 \mathrm{mOsm} / \mathrm{kg})$. Slices were kept in room temperature ACSF until ready to measure and were used within $8 \mathrm{~h}$. All solutions were bubbled with carbogen $\left(95 \% \mathrm{O}_{2}, 5 \% \mathrm{CO}_{2}\right)$ for the duration of the preparation and subsequent experiment.

For imaging, slices were mounted on Poly-L-Lysine (PLL)-coated coverslips. Coverslips (Fisher, 12-545-80) were plasma cleaned for $3 \mathrm{~min}$, covered with 50-100 $\mu \mathrm{l} 0.1 \%$ (w/v) PLL (150-300 kDa) solution (Sigma-Aldrich, P8920) and allowed to dry under vacuum. Coverslips were thoroughly washed with nanopore water and dried before use. To mount the tissue, a slice was transferred to the PLL-coated face of the coverslip with a Pasteur pipette. Excess ACSF was pipetted or wicked away with filter paper in the process flattening out the brain slice and adhering it to the glass. We found that this method worked reliably for coronal slices from one hemisphere but not for coronal slices from the entire brain. Coverslips were placed in a custom-built flow chamber with a microscope slide bottom and \#1.5 coverslip lid. ACSF was perfused at a rate of $1 \mathrm{ml} / \mathrm{min}$ with a VWR peristaltic pump.

The imaging protocol consisted of a $2 \mathrm{~s}$ imaging epoch followed by a $400 \mathrm{~ms}$ stimulation period and another $2 \mathrm{~s}$ imaging epoch. Each imaging epoch comprised 11 frames of functional Hadamard acquired with a 180 $\mathrm{ms}$ period under $100 \mathrm{~mW} / \mathrm{cm}^{2} 561 \mathrm{~nm}$ illumination. Blue light stimula- 
tion protocols are described in figure captions. The slice was allowed $6 \mathrm{~s}$ to recover before starting another imaging epoch. One run consisted of six imaging and stimulation rounds over $1 \mathrm{~min}$. Runs were repeated several times, spaced out by at least $5 \mathrm{~min}$. NBQX and CPP, or tetrodotoxin (TTX; Tocris Bioscience) or retigabine, phenytoin, or carbamazepine (Sigma-Aldrich) were added to the ACSF from $1000 \times$ stock solutions after several baseline runs. In Figure 4, brain slices were randomly selected from a pooled set of slices for different drug treatments.

\section{Analysis of slice data}

Registration. After reconstruction of Hadamard images (see Materials and Methods), frames for each epoch were averaged together. Small movements and deformations in the slice over the course of multiple runs were corrected by automatic non-rigid registration (Klein et al., 2010). Functional Hadamard recording and structural Hadamard images were manually registered using a $2 \mathrm{D}$ affine transformation.

Cell selection. $\Delta \mathrm{F}$ images were calculated for each registered run by subtracting images acquired before blue light stimulation from images acquired immediately after blue-light stimulation. Peaks in $\Delta \mathrm{F}$ images corresponded to individual cells, but noise in $\Delta \mathrm{F}$ varied as a result of brightness inhomogeneities in the slice, making it difficult to extract peaks directly. To correct for this noise, a wide-field image for each slice was blurred with a 2D Gaussian with an 8 pixel $(19.2 \mu \mathrm{m}) \mathrm{SD}$, to remove nucleus sized objects. The square root of this image was used to normalize the $\Delta \mathrm{F}$ image of the slice. High spatial frequency noise was removed with a $2 \mathrm{~d}$ Gaussian filter with a 0.5 pixel $(1.2 \mu \mathrm{m}) \mathrm{SD}$. Regions without expression were manually selected and SDs in these regions were chosen as a noise floor. Cells were identified as peaks in the normalized $\Delta \mathrm{F}$ image, which had an amplitude larger than the noise floor by a userdefined factor, typically $7-10$. Cells were required to have a minimum distance in space of 4 pixels $(9.6 \mu \mathrm{m})$ to avoid double counting cells. Once cell locations were identified, single-cell fluorescence traces were extracted from corresponding locations in movies of un-normalized data blurred with 2d Gaussian filter with 1 pixel SD.

Exclusion of spontaneously active and dying cells. While measuring a large number of cells in an acute slice, a portion of cells showed spontaneous activity, characterized by transient fluorescent increases uncorrelated with blue light stimulation; and cell death, characterized by a large and irreversible increase in fluorescence. For Figures 6-9, slices were imaged nine times, five times before antiepileptic drug (AED) application and four times after. Imaging epochs were averaged to generate movies with 108 frames ( 12 epochs per run $\times 9$ runs). After extracting cell traces from these movies for all slices in the experiment, each cell's mean and SD per run were calculated. Least-squares fit on the mean and SD were performed on three pre-drug runs and projected to the full nine runs. Cells were excluded from further analysis if any projected mean or $\mathrm{SD}$ was $<1 / 15$ of the cell's mean value or if the root mean square error of the fit was larger than $1 / 15$ of the cell's mean value. This procedure rejected $<17 \%$ of cells.

Generation of excitability maps. To generate the maps in Figure 7 the fluorescence trace for each included cell was normalized by subtracting its mean fluorescence values for each run and normalizing by the SD for each run. For each cell, three pre-drug runs were averaged together to yield a 12 element vector corresponding to normalized $F$ in each epoch. Principal component analysis (PCA) yielded three main principal components, which were then back-projected into pixel space for each slice, yielding the black and white images in Figure $7 c$. Color images (Fig. $7 d, e$ ) were generated using $\mathrm{L}^{*} \mathrm{a}^{\star} \mathrm{b}$ colorspace, by projecting PC1 into lightness, $\mathrm{L}$, and PC2 and PC3 into the red-green and blue-yellow axes, $a$ and $b$.

To generate maps of changes in drug response in Figure $8 a, \Delta \mathrm{F}$ images from four runs before and after drug addition were averaged together, median filtered with a 3 pixel kernel, saturated at their 99.5 percentile, and displayed in the green and red channels, respectively. The blue channel is the average of the red and green images. Color saturation was adjusted in $L^{*} a^{\star} b$ space to aid in visualization. In Figure $9, \Delta F$ images are scaled to the same absolute counts and shown in separate color channels.

Cortical layer analysis. All striatal cells were pooled and treated separately. For cortical cells, cortex boundaries were manually defined in structural images as the surface of the brain and the bottom of layer 6 .
Boundaries were registered to functional images and cells were assigned a normalized depth coordinate based on these boundaries. Drug response, defined as $\Delta \mathrm{F}_{\mathrm{drug}} / \Delta \mathrm{F}_{0}$, could then be related to normalized cortical depth. For each slice, cells were binned by cortical depth and the drug response per cell averaged over cells. Extreme cell responses were excluded from each bin using the generalized extreme Studentized deviate test. Layer boundary locations were taken from the primary somatosensory cortex in the matched coronal slices of the Allen Brain Reference Atlas.

KCNQ3 expression levels were acquired from Allen Brain Institute experiment 100041071 . The somatosensory cortex was manually defined in 11 sagittal slices from a P28 male mouse. The available expression image was used to mask the raw data, but expression values were obtained directly from the raw ISH data. The edges of the cortex and cortical depth bins were defined as described and expression values were averaged together across slices from the same experiment.

\section{Results}

\section{A spectrally orthogonal $\mathrm{Ca}^{2+}$ sensor and channelrhodopsin for $1 P$ AON}

AON requires a spectrally orthogonal optogenetic actuator and activity reporter (Fig. 1a). Examination of channelrhodopsin action spectra and $\mathrm{Ca}^{2+}$ reporter excitation spectra suggested that the best approach for 1P AON was to use a blue-shifted channelrhodopsin and a RGECI (Fig. 1a). We thus set out to identify protein pairs suitable for this purpose.

We began by comparing the single action potential responses of RGECIs in cultured neurons. jRGECO1a was the most sensitive $(\Delta \mathrm{F} / F=54 \pm 10 \%, n=\sim 120$ neurons. Unless otherwise indicated, all uncertainties are SEM), followed by R-CaMP2 and jRCaMP1a, consistent with previous reports (Fig. $1 b$; Table 1; Inoue et al., 2015). R-CaMP2 had the fastest kinetics ( $\tau_{\text {on }}=26 \pm$ $10 \mathrm{~ms}, \tau_{\text {off }}=270 \pm 20 \mathrm{~ms}, n=\sim 120$ neurons), followed by jRGECO1a $\left(\tau_{\text {on }}=47 \pm 1 \mathrm{~ms}, \tau_{\text {off }}=440 \pm 40 \mathrm{~ms}, n=\sim 120\right.$ neurons) and jRCAMP1a (Fig. 1c, Table 1). In HEK293T cells, under basal $\mathrm{Ca}^{2+}$ conditions, jRGECOla had the longest photobleaching time constant $\left(\tau_{\text {bleach }}=81 \pm 5 \mathrm{~s}, I_{561}=44 \mathrm{~W} / \mathrm{cm}^{2}, n=\right.$ 9 cells), followed by R-CaMP2 and jRCaMP1a (Table 1). Under typical imaging conditions $\left(I_{561}=0.1 \mathrm{~W} / \mathrm{cm}^{2}\right)$, photobleaching of $j$ RGECO 1 a was thus $<10 \%$ during $1 \mathrm{~h}$ of continuous imaging. Although photobleaching is often a concern for $1 \mathrm{P}$ imaging, these results established that this effect was minor for wide-area imaging of jRGECO1a. We selected jRGECO1a for its superior sensitivity and photostability.

mApple-based fluorescent sensors, including jRGECO1a, are known to undergo photoswitching under blue light illumination (Akerboom et al., 2013; Dana et al., 2016). We thus sought a blue-shifted channelrhodopsin that could drive spikes in jRGECO1a-expressing neurons at blue intensities low enough to avoid optical crosstalk. TsChR is the most blue-shifted published ChR (Fig. 1a), but was initially reported to produce only $\sim 40 \%$ as much photocurrent as ChR2(H134R); (Klapoetke et al., 2014) and so has not previously been used in optogenetic applications. Addition of a $\mathrm{K}_{\mathrm{ir}} 2.1$ trafficking sequence (TS) and a GFP expression tag to TsChR led to excellent trafficking in cultured neurons (Fig. 1d). We called this construct eTsChR-eGFP. Compared with ChR2(H134R), eTsChR had higher steady-state photocurrents $(470 \pm 42$ vs $288 \pm 60 \mathrm{pA}, p=0.034$, Student's $t$ test, $n=6$ neurons each; Fig. 1e). At the highest blue-light intensity tested $\left(33 \mathrm{~W} / \mathrm{cm}^{2}\right), \mathrm{ChR} 2(\mathrm{H} 134 \mathrm{R})$ passed a steady-state photocurrent of $288 \pm 60 \mathrm{pA}$; eTsChR passed the same steady-state photocurrent at 100 -fold lower intensity $\left(0.33 \mathrm{~W} / \mathrm{cm}^{2}\right)$. Compared with ChR2(H134R), eTsChR also had higher maximum steady-state photocurrent densities $(13.2 \pm 1.2 \mathrm{pA} / \mathrm{pF}$ vs $7.8 \pm 2.0 \mathrm{pA} / \mathrm{pF}, p=$ 

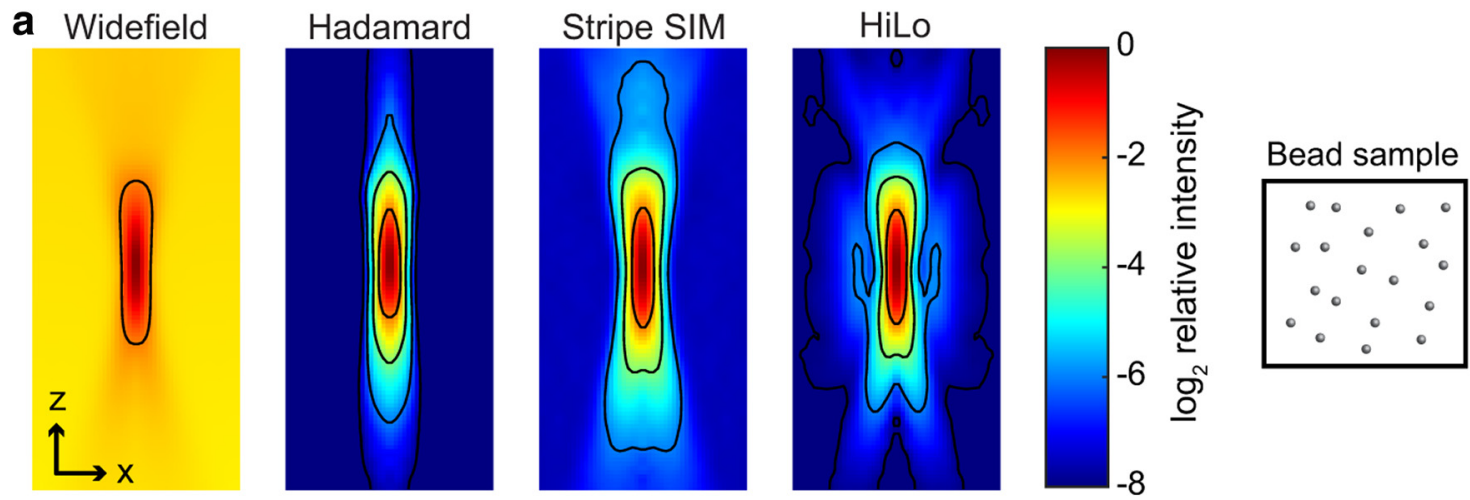

$\mathrm{Z}=0 \mu \mathrm{m}$
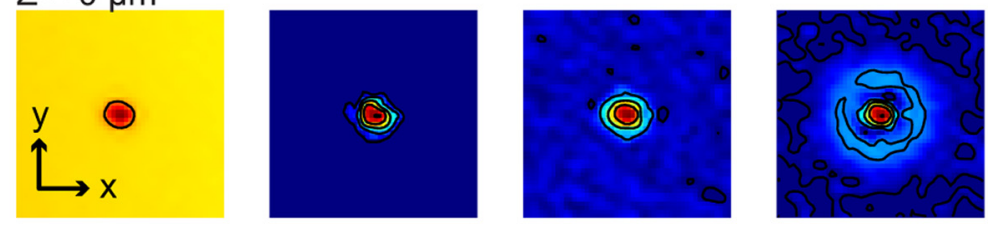

$Z=+30 \mu \mathrm{m}$
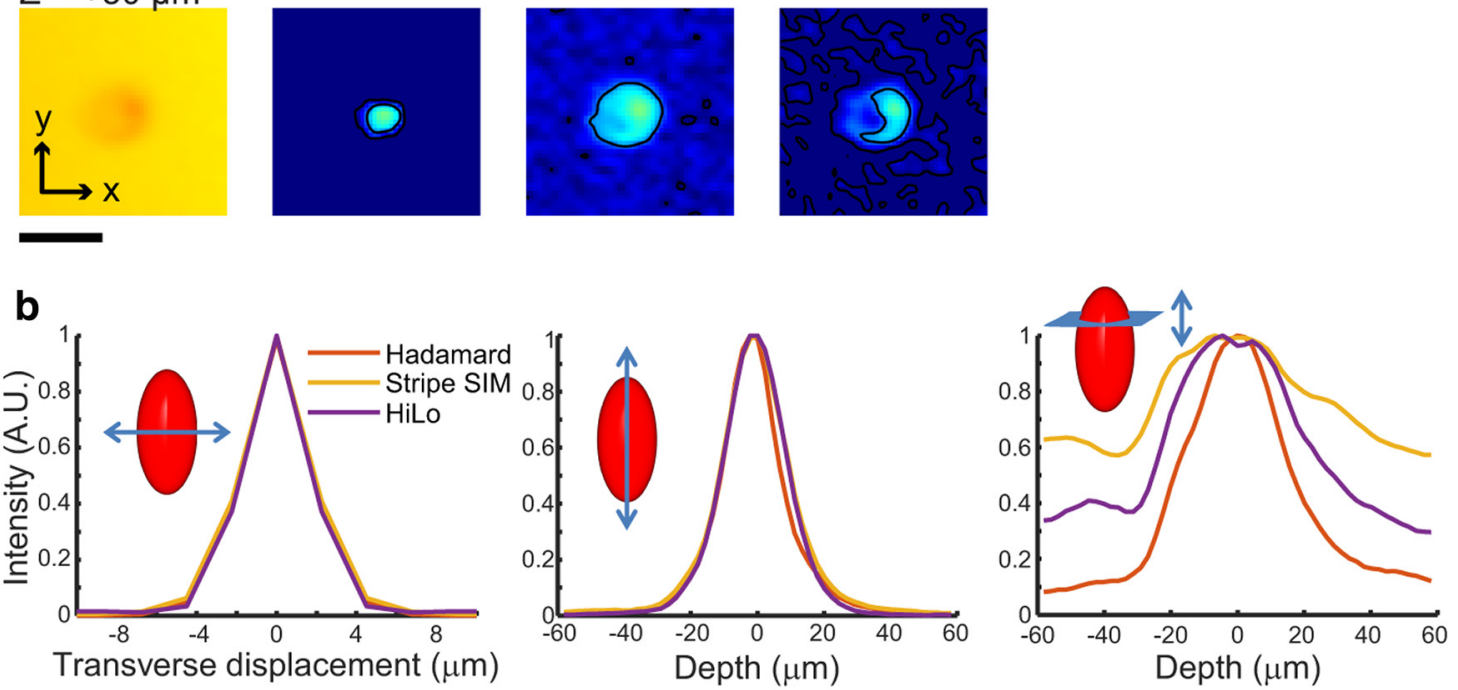

C
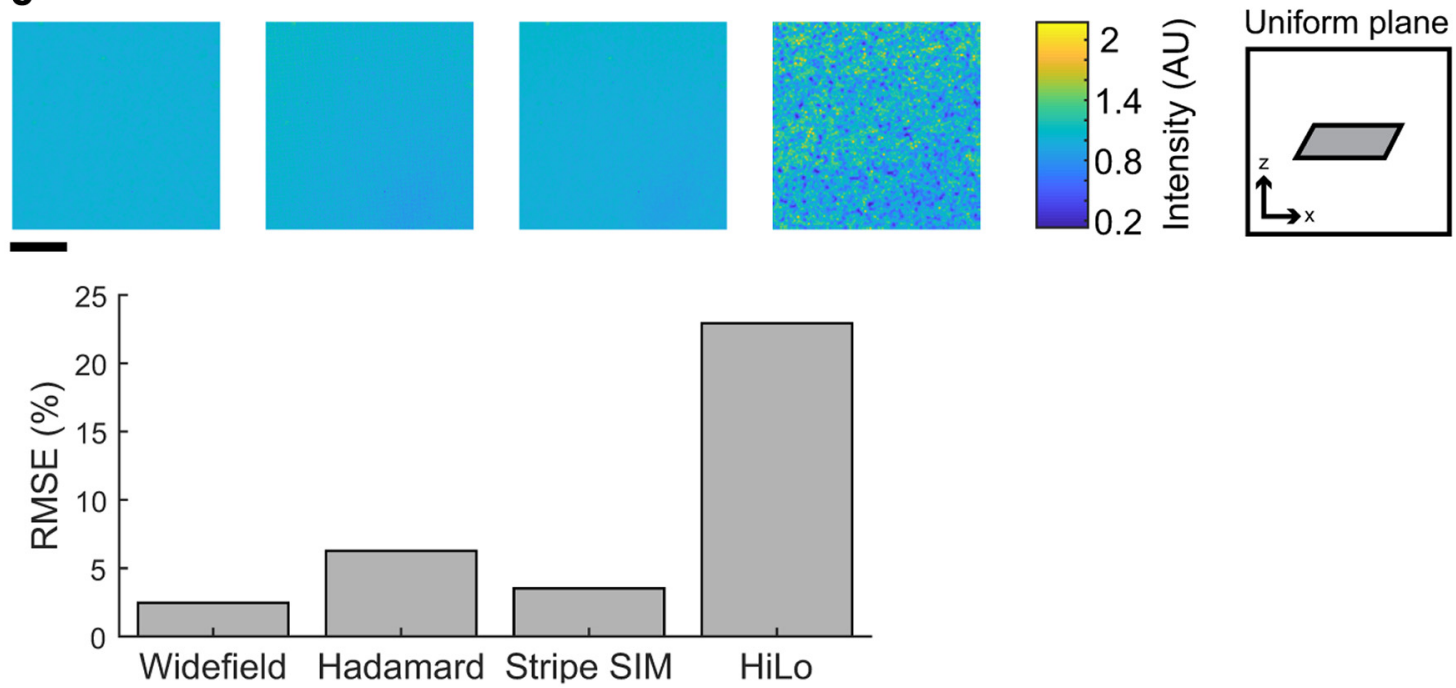

Figure 4. Characterization of Hadamard microscopy. $\boldsymbol{a}, \boldsymbol{b}$, Comparison of SIM optical sectioning methods using subdiffraction beads. $\boldsymbol{a}$, Images show (left to right): wide-field epifluorescence, Hadamard microscopy using 12 patterns, stripe SIM with period 4 pixels and four phases, and HiLo microscopy using DMD-projected pseudorandom patterns. Top row, Radially averaged meridional cross-section of the PSF. Second row, Transverse cross-section at the focal plane. Third row, Transverse cross-section at $30 \mu \mathrm{m}$ defocus. For all $\boldsymbol{a}$ panels, the color scale is logarithmic, and contours were drawn on every fourfold change in intensity. Scale bar, $20 \mu \mathrm{m}$, isotropic. $\boldsymbol{b}$, Left and center, Lateral and axial line profiles through the PSF show equivalent (Figure legend continues.) 
0.044 , Student's $t$ test, $n=6$ ) and faster on- and off-kinetics (Fig. $1 f, g)$.

We coexpressed jRGECO1a and eTsChR in cultured rat hippocampal neurons, and used the far-red voltage-sensitive dye BeRST1 (Huang et al., 2015) as a ground-truth reporter of neural spiking. Flashes of blue light $\left(0.7 \mathrm{~W} / \mathrm{cm}^{2}, 10 \mathrm{~ms}\right)$ induced action potentials, reported by BeRST1 fluorescence, and $\mathrm{Ca}^{2+}$ transients, reported simultaneously by jRGECO1a fluorescence (Fig. $1 h)$. The sodium channel blocker TTX $(1 \mu \mathrm{M})$ eliminated the light-evoked transients in both the BeRST1 and jRGECO1a fluorescence channels, confirming that the jRGECOla response reflected spiking-dependent $\mathrm{Ca}^{2+}$ influx and that the optogenetic stimulation did not induce detectable photo-artifacts in the jRGECO1a fluorescence.

Cytoplasmic expression of jRGECOla in brain slices led to a high level of fluorescence background from reporter present in neuropil, even with the optical sectioning approach described in this work (Fig. 2a). To facilitate imaging in tissue, we fused jRGECOla to a Histone-2B (H2B) tag to localize expression to the nucleus (Figs. $1 d$, $2 b$ ), as previously done for zebrafish (Freeman et al., 2014) and rat (Skocek et al., 2018) brain imaging. The nuclear-localized H2BjRGECOla showed clearly resolved nuclei with little background between the cells. In cultured neurons, H2B-jRGECO1a responded to single action potentials with good sensitivity $(\Delta \mathrm{F} / F=19.4 \pm$ $5.3 \%, n=3$ cells), but with slower kinetics than the cytosolic reporter $\left(\tau_{\text {on }}=167 \pm 27 \mathrm{~ms}, \tau_{\text {off }}=1400 \pm 270 \mathrm{~ms}\right)$ consistent with previous measurements of nuclear $\mathrm{Ca}^{2+}$ dynamics (Fig. $2 c$; Eder and Bading, 2007; Bengtson et al., 2010).

We tested for optical crosstalk between actuator and reporter channels in cells coexpressing the optimized AON constructs. Because of the high sensitivity of eTsChR, the blue light doses needed to elicit spikes $\left(0.7 \mathrm{~W} / \mathrm{cm}^{2}\right.$ for $\left.10 \mathrm{~ms}, \lambda=488 \mathrm{~nm}\right)$ induced minimal photoartifact in either cytoplasmic or nuclear jRGECO1a compared with a single-spike $\mathrm{Ca}^{2+}$ signal (Fig. $2 d$, $-2 \%$ photoartifact vs $1 h, 2 e, 19 \%$ spike response). Crosstalk from direct blue-light excitation of jRGECO1a fluorescence was avoided in our experiments by interleaved optogenetic stimulation and fluorescence imaging.

The yellow light used for $\mathrm{Ca}^{2+}$ imaging $(\lambda=561 \mathrm{~nm}, 0.1$ $\mathrm{W} / \mathrm{cm}^{2}$ ) induced in eTsChR a steady-state photocurrent $<0.5 \mathrm{pA}$ (Fig. 2f), far too small to trigger spurious action potentials. Expression of eTsChR did not significantly affect neurons' membrane resistance, membrane capacitance, or resting potential compared with controls (Table 2). Together, eTsChR and H2BjRGECO1a formed a suitable actuator/reporter pair for crosstalkfree $1 \mathrm{P} A O N$.

\section{$\leftarrow$}

(Figure legend continued.) resolution for the three sectioning methods. Right, Integrated intensity in transverse cross-sections reveals off-axis spurious side lobes in stripe SIM and HiLo which contribute to out-of-focus crosstalk. c, A uniform fluorescent plane at the focal plane resulted in larger inhomogeneities when imaged using HiLo in comparison with the other methods, a consequence of inhomogeneities in HiLo illumination (Mazzaferri et al., 2011). The fractional noise in HiLo did not decrease with increasing photon counts. Top, Optical section images of the uniform plane, all shown at the same linear color scale. Bottom, Deviations from uniformity in the images on top. Hadamard and stripe SIM microscopies avoided this artifact by providing illumination whose time-average intensity was precisely the same at all sample points. Scale bar, $200 \mu \mathrm{m}$. For all panels, each sample was imaged in matched conditions for all methods (number of images, illumination intensity, acquisition time). Detailed description in Materials and Methods.
Table 2. Patch characterization of eTsChR

\begin{tabular}{lrcl}
\hline & eTsChR & ChR2(H134R)-GFP & $\begin{array}{l}\text { Pvalue, } \\
\text { Student's } t \text { test }\end{array}$ \\
\hline Access resistance, $\mathrm{M} \Omega$ & $12.3 \pm 1.5$ & $12.4 \pm 1.3$ & 0.96 \\
Membrane resistance, $\mathrm{M} \Omega$ & $633 \pm 84$ & $467 \pm 88$ & 0.20 \\
Membrane capacitance, $\mathrm{pF}$ & $36.5 \pm 4.8$ & $44.9 \pm 9.7$ & 0.45 \\
Resting potential, $\mathrm{mV}$ & $-36.5 \pm 4.8$ & $-44.3 \pm 2.9$ & 0.13 \\
\hline
\end{tabular}

Patch parameters of cells in Figure 1. All values are reported as mean $\pm \mathrm{SEM} ; n=6$ cells throughout.

\section{Hadamard microscopy enables optical sectioning in ultra- wide-field images of acute brain slices}

We next sought to perform wide-area optically sectioned imaging of the AON constructs in acute brain slices. To achieve high light collection efficiency over a wide FOV, we designed a microscope system around a low-magnification high numerical aperture objective (Olympus, MVPLAPO 2 XC, NA 0.5). In wide-field epifluorescence mode, this microscope imaged a $4.6 \mathrm{~mm}$ FOV, large enough to capture most of a hemisphere of a coronal brain slice, with nominal $2.25 \mu \mathrm{m}$ lateral resolution set by the pixel size on the sCMOS detector. Apart from the optical filters and the mechanical mounts, all elements of the microscope were off-theshelf components (see Materials and Methods).

To achieve optical sectioning over a wide FOV, we developed a structured illumination approach based on Hadamard encoding. We placed a DMD in the illumination path to enable arbitrary spatiotemporal patterning of the fluorescence excitation. Each DMD pixel mapped to $6.3 \mu \mathrm{m}$ in the sample plane. The DMD modulated the excitation light with a series of binary illumination patterns such that neighboring sample locations were illuminated with orthogonal intensity sequences (Fig. $3 a, P_{1}$, $\left.P_{2}, \ldots, P_{\mathrm{n}}\right)$. Raw data consisted of a series of images [Fig. $3 b, 1$ : $\left.F\left(t_{1}\right), F\left(t_{2}\right), \ldots, F\left(t_{\mathrm{m}}\right)\right]$ acquired with each illumination pattern, which were then demodulated to yield images of the scattered light for each illumination location (Fig. 3b, 2). Software binary masks then rejected scattered light (Fig. 3b, 3), akin to physical pinholes used in confocal microscopy. The sum of images over all illumination locations yielded an optical section (Fig. 3b, 4; see Materials and Methods). The Hadamard algorithm is linear and local, i.e., the image resulting from two distinct sources is the sum of the images of the individual sources; and the final intensity value at each pixel depends only on signals acquired at that pixel. Thus the results are independent of the sample and do not require any specialized post-processing.

To make all projected DMD pixels mutually orthogonal would require prohibitively long digital codes $\left(\sim 10^{6}\right.$ samples $)$, but because light scatter is mostly local, repeating the codes periodically at separations larger than the scattering point-spread function resulted in minimal crosstalk (Fig. 3c). Residual crosstalk between repeated codes was scrambled by inverting the sequence of a randomly selected 50\% subset of the pixels (Fig. 3c; see Materials and Methods). This procedure resulted in series of patterns with $50 \%$ duty cycle, uniform mean illumination across the sample, and uniform spatial and temporal spectral density. By varying the number of frames in the Hadamard sequence, one can systematically trade time resolution versus background rejection. The workflow for acquiring and analyzing Hadamard images is summarized in Figure 3d. A link to the software is given in Materials and Methods. Application of compressed sensing algorithms to Hadamard microscopy enabled signal extraction at half the frame rate of the camera (Parot et al., 2019; see Fig. 10), though this improved time resolution was not required for the applications described in this work. 
a
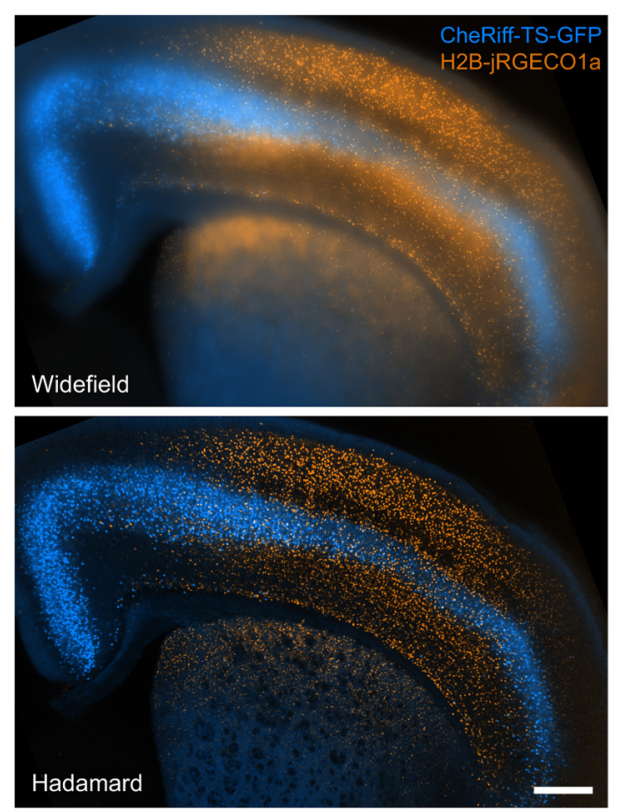

b

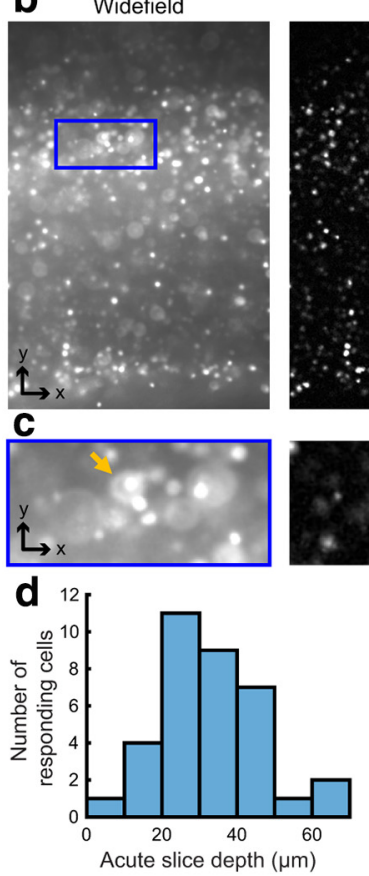

Hadamard
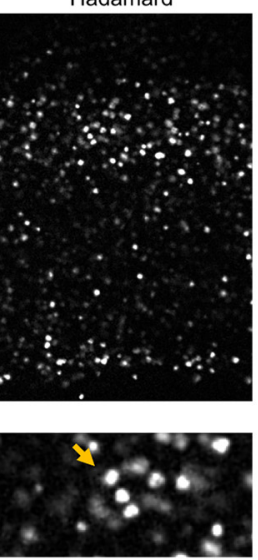

e

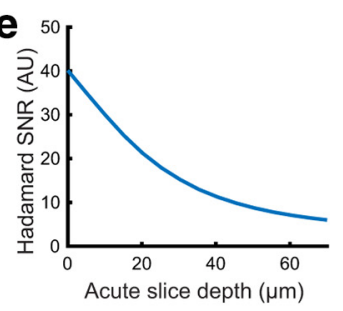

SIM
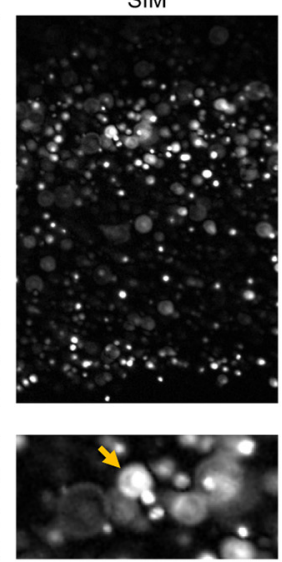

HiLo
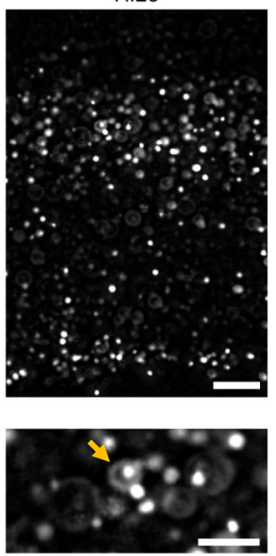

$f$

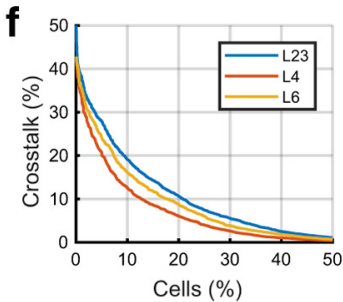

Figure 5. Hadamard microscopy resolves individual H2B-jRGEC01a-labeled neurons in acute brain slices. $\boldsymbol{a}$, Two-color fluorescence maximum-intensity projections acquired from an acute brain slice expressing H2B-jRGEC01a broadly in cortex and striatum and membrane targeted CheRiff-TS-GFP in L5 pyramidal cells. Top, Wide-field epifluorescence. Bottom, Hadamard image. Scale bar, $500 \mu \mathrm{m} . \boldsymbol{b}$, Images acquired with different 1P computational optical sectioning methods. Images were acquired in the same sample with matched conditions (number of images, illumination intensity, acquisition time). The sample comprised an acute brain slice expressing H2B-jRGEC01a. Images show (left to right): wide-field epifluorescence, Hadamard microscopy using 12 patterns, SIM with period 4 pixels and four phases, and HiLo microscopy using DMD-projected speckle patterns. Scale bar, $100 \mu \mathrm{m}$. The blue box region is expanded in c. c, Hadamard microscopy avoids defocus lobes present using other methods. The orange arrows indicate a defocused cell that is rejected by Hadamard microscopy but appears in the other techniques. All images use the same linear scale of normalized gray values. Scale bar, $50 \mu \mathrm{m}$. $\boldsymbol{d}$, Depth distribution of responsive cells during Hadamard functional recording, measured by high resolution confocal microscopy acquired after the functional measurement and registered to the Hadamard images. The depth was $32.2 \pm 12.7 \mu \mathrm{m}$ (mean \pm SD, $n=35$ neurons). $\boldsymbol{e}$, Depth-dependent decay in SNR for Hadamard microscopy in acute slices. Decay length was $\sigma_{z}=27 \mu \mathrm{m}$. $\boldsymbol{f}$, Estimated distribution of crosstalk in neuronal recordings using Hadamard microscopy. 0 nly $10 \%$ of cells had $>20 \%$ crosstalk (fluorescence attributable to other cells) in L2/3 (see Materials and Methods).

We compared Hadamard microscopy to two other SIM techniques, stripe SIM (Gustafsson, 2000) and HiLo (Mertz and Kim, 2010), all implemented using the same DMD and optics. Images of $0.2 \mu \mathrm{m}$ fluorescent beads in agarose were used to estimate the PSFs of the three techniques in a non-scattering medium. As, expected, line sections through the three PSFs gave identical lateral (FWHM $2.7 \mu \mathrm{m}$ ) and axial (FWHM $14.0 \mu \mathrm{m}$ ) resolution near the focus (Fig. 4). For the low-magnification, wide-area implementation described here, the resolution in all three cases was determined by the intersection of the pixel-size-limited DMD illumination spots and the camera collection PSFs. We performed optical simulations to explore whether lateral shifts between DMD pixels and the smaller camera pixels would lead to spatially varying spatial resolution. For the parameters of our experimental setup, the changes in spatial resolution were $<5 \%$ in lateral resolution and $<10 \%$ in axial resolution, so these effects were subsequently neglected.

The three imaging techniques differed critically in imaging parameters not captured by the FWHM of the PSFs, however. Stripe SIM and HiLo PSFs had out-of-focus conical lobes, a consequence of out-of-focus points emitting along the same rays as in-focus and laterally offset points. These lobes did not lie along either the lateral or axial line sections through the PSF, so they did not contribute to the PSF dimensions as usually characterized, but they contributed to substantial out-of-plane total fluorescence (Fig. 4b). Hadamard images lacked this artifact because use of multiple illumination patterns resolved ambiguities in assignment of out-of-focus fluorescence. For Hadamard microscopy, the integrated the PSF in the transverse $(x-y)$ plane decayed to $15 \%$ of its peak at a defocus of $-30 \mu \mathrm{m}$, whereas by the same measure HiLo retained $38 \%$ of peak fluorescence and stripe SIM retained $62 \%$ of peak fluorescence (Fig. $4 b$ ). Thus HiLo and stripe SIM suffered $\sim 2.5$-fold and $\sim 4$-fold higher background than Hadamard, respectively.

For the purpose of rejecting out-of-focus background fluorescence in tissue, the integrated transverse fluorescence, not the more commonly used axial line section, is the critical parameter. Thus we expected that Hadamard microscopy would perform better than stripe SIM or HiLo in resolving single-cell signals in densely expressing tissues. Figure $4 c$ and the Discussion compare the technical noise and shot noise properties of Hadamard and other SIM techniques. Hadamard performed as well as or better than the other techniques by these parameters.

We compared the performance of the three structured illumination techniques in brain tissue (Fig. 5). The sample comprised an acute $300-\mu \mathrm{m}$-thick coronal brain slice, expressing nucleartargeted jRGECO1a throughout cortex and striatum, and membrane-targeted CheRiff-GFP restricted by an Rbp4-Cre driver to a subset of layer (L) 5 pyramidal cells (Fig. $5 a$ ). Hadamard images clearly resolved individual cells, whereas wide-field epifluorescence did not (Fig. 5b). In the stripe SIM and HiLo images, out of focus nuclei appeared as bright rings, a consequence of the conical lobes on the PSF, which prevented clear separation of single-cell images (Fig. $5 c$ ). Light scattering caused the Hadamard signal to decay as a function of image depth with a length constant of $27 \mu \mathrm{m}$ in acute brain slices (Fig. 5d,e) and 113 
$\mu \mathrm{m}$ in fixed slices. The difference in signal attenuation was attributed to decreased light scattering after the fixation process.

To quantify the ability of Hadamard microscopy to resolve single-cell signals, we used high-resolution confocal microscopy to make ground-truth maps of the spatial distribution of nuclei in fixed slices densely expressing nuclear jRGECO1a. We then simulated Hadamard images of these cells in scattering tissue and estimated the crosstalk, i.e., the spurious contribution from all other cells to the fluorescence signal ascribed to each nucleus (see Materials and Methods). In cortical layer 2/3, only $10 \%$ of the cells received $>20 \%$ crosstalk from other cells. The crosstalk was lower in other brain regions (Fig. 5f). Cell nuclei had a stereotyped round and localized shape. The degree of crosstalk could be estimated on a cell-by-cell basis via shape deviations. If desired, cells with crosstalk beyond a threshold value could be discarded from the analysis, though this procedure was not used here. Hadamard microscopy thus enabled optically sectioned imaging with single-cell resolution over wide FOVs in acute brain slices.

\section{Mapping excitability in acute slices}

To map neural excitability, we applied Hadamard microscopy with simultaneous optogenetic stimulation in acute mouse brain slices expressing the actuator-reporter pair. We coinjected AAV9-hSynDO-H2B-jRGECOla and AAV9-hSyn-eTsChR in cortex and striatum of wild-type $\mathrm{P} 0-\mathrm{P} 2$ mouse neonates (Fig. 6a). Both proteins expressed well and were readily visualized via Hadamard imaging in $300 \mu \mathrm{m}$ acute brain slices from 3-week-old animals (Fig. $6 b, c)$. We performed Hadamard AON measurements in a region $2.3 \times 2.3 \mathrm{~mm}$, set by the size of the expressing region. Cell signals were acquired from a depth of $32 \pm 13 \mu \mathrm{m}$ (Fig. $5 d$ ).

To probe excitability, we exposed the slice to a series of widefield blue stimuli of increasing strength, interleaved with Hadamard imaging of H2B-jRGECO1a with yellow light $(561 \mathrm{~nm}$, $100 \mathrm{~mW} / \mathrm{cm}^{2}$; Fig. 6d). Hadamard images were first acquired for $2 \mathrm{~s}$ to establish baseline fluorescence. Then a brief burst of blue light pulses ( $470 \mathrm{~nm}, 8$ pulses, $15 \mathrm{~mW} / \mathrm{cm}^{2}, 5 \mathrm{~ms}$ duration, $20 \mathrm{~Hz}$ ) evoked neural activity, followed by another $2 \mathrm{~s}$ of Hadamard imaging to record the response. This image-stimulate-image procedure was repeated at $10 \mathrm{~s}$ intervals, six times, with the intensity of the blue light doubling upon each repetition to a maximum of $480 \mathrm{~mW} / \mathrm{cm}^{2}$. This measurement protocol reported the changes in intracellular $\mathrm{Ca}^{2+}$ concentration as a function of optogenetic stimulus strength.

Neighboring cells often showed distinct patterns of $\mathrm{Ca}^{2+} \mathrm{dy}-$ namics, whereas interstitial regions showed undetectable fluorescence (Fig. 6d,e), confirming that Hadamard microscopy effectively rejected scatter and out-of-focus background. The yellow light used for $\mathrm{Ca}^{2+}$ imaging induced spurious activity in only $0.46 \pm 0.03 \%$ of cells ( $n=38,835$ cells, 9 slices), establishing that the imaging light only weakly activated eTsChR. The sodium channel blocker TTX $(1 \mu \mathrm{M})$ abolished blue light-evoked responses slice wide, confirming that $\mathrm{Ca}^{2+}$ responses were because of action potential firing (Fig. $6 f, g$ ) and, furthermore, that blue light-induced photoswitching was minimal.

We tested the long-term stability of the preparation. The optogenetically induced $\mathrm{Ca}^{2+}$ signal was stable over a 78 min session comprising 7 repeated imaging cycles (Fig. $6 h, i$ ). During this period the population-average optically evoked $\Delta \mathrm{F} / \mathrm{F}$ at the strongest stimulus decreased modestly from $64 \pm 0.7 \%$ to $52 \pm$ $0.7 \%, n=3,195$ cells. These results demonstrate the capability for repeated measurements over $>1 \mathrm{~h}$ in a single sample.

We used a 2D peak-finding algorithm to identify $n=6,102$ responding cells in the Hadamard images of a single brain slice
(Fig. 7a). Cells showed different patterns of response in the striatum versus cortex, but we also observed cell-to-cell variability within the cortex. To characterize this variability, we applied PCA to a set of single-cell recordings. First, we repeated the excitability measurement on 9 slices from 2 animals, recording from a total of $n=32,103$ cells across cortex and striatum. Measurement runs (comprising 6 measure-stimulate-measure sequences) were repeated at $5 \mathrm{~min}$ intervals, three times per slice. PCA identified three main temporal components in the single-cell fluorescence responses (Fig. 7b,c; see Materials and Methods). Examination of the PC temporal waveforms showed that PC1 measured overall fluorescence response amplitude, PC2 captured a left-right shift in the sigmoidal excitability profile, and PC3 largely captured a stimulus-dependent increase in baseline fluorescence.

We then decomposed the fluorescence waveform at each pixel into its principal components (PCs), and color-coded each pixel by its PC amplitudes (Fig. $7 d$,e; see Materials and Methods). Despite coloring each pixel independently, individual cells appeared homogeneously colored in the resulting image (Fig. 7e), consistent with the low cell-to-cell fluorescence crosstalk. These maps revealed striking colored bands running along the cortical layers, demonstrating different functional responses in different brain regions. Intriguingly, some layers appeared relatively homogeneous (L2/3, L4, L6), whereas cells in L5 had larger cell-to-cell variations in response. These results demonstrate that Hadamard AON can map excitability over thousands of individual neurons across large areas of acute brain slice.

\section{Mapping pharmacological responses with Hadamard AON}

Wide-area AON offers a means to map the cell type and regionspecific effects of pharmacological or other perturbations on neural excitability. We performed excitability measurements on acute slices before and after applying the AEDs retigabine (25 $\mu \mathrm{M})$, carbamazepine $(100 \mu \mathrm{M})$, and phenytoin $(100 \mu \mathrm{M})$. To quantify the drug effect, we measured the pixel-by-pixel change in mean amplitude, $\Delta \mathrm{F}$, of the optogenetically induced response; a parameter close to the first principal component that emerged from the unsupervised analysis. Each drug had different effects in striatum and cortex, and attenuated cortical excitability in a distinctive spatial pattern (Fig. $8 a$ ).

We sorted cells into bins based on their cortical depth and visualized mean AED response as a function of cortical depth, averaged over $n=3$ slices per drug (Fig. 8b). Carbamazepine and phenytoin, both sodium channel blockers, showed relatively uniform suppression of excitability as a function of cortical depth, but retigabine showed a graded response, weakest in L6b and strongest in L4.

Retigabine is a specific positive allosteric modulator of $\mathrm{K}_{\mathrm{v}} 7$ channels, and its primary target is thought to be the $\mathrm{K}_{\mathrm{v}} 7.2 / 7.3$ heteromer (Gunthorpe et al., 2012), coded for by the genes KCNQ2 and KCNQ3. We examined the Allen Brain Atlas map of the expression level of KCNQ3 (Lein et al., 2007), as determined by RNA in situ hybridization (ISH), and found statistically significant correlation between KCNQ3 expression level and effect of retigabine (Pearson's $r=-0.40,95 \%$ confidence interval between -0.022 and -0.69 obtained by bootstrapping; Fig. $8 c, d)$. Higher expression of KCNQ3 correlated with greater inhibition of excitability by retigabine, as one would expect for a potassium channel activator. An independent ISH study in adult animals reported a similar distribution of KCNQ2 and KCNQ3 (Saganich et al., 2001). These results establish a connection between the Hadamard AON measurements and the underlying pattern of ion channels. 
a

hSyn-eTsChR + hSyn-DO-H2B-jRGECO1a

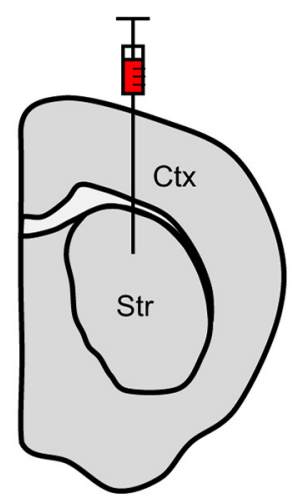

b

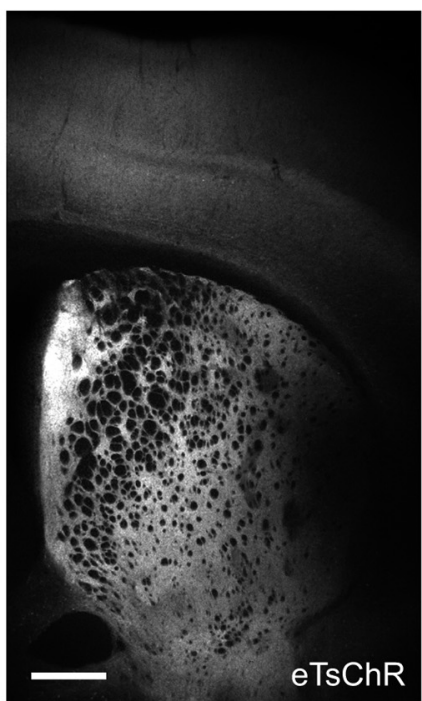

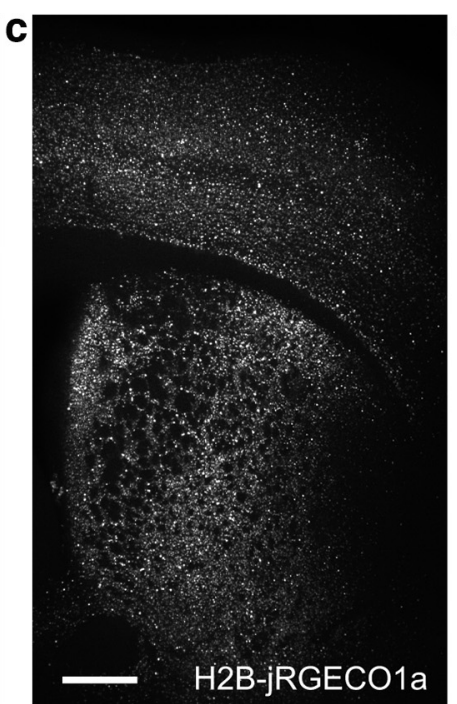

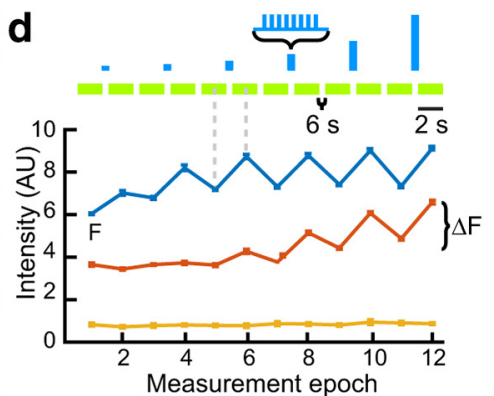

e

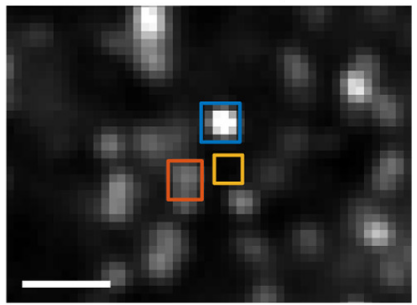

f

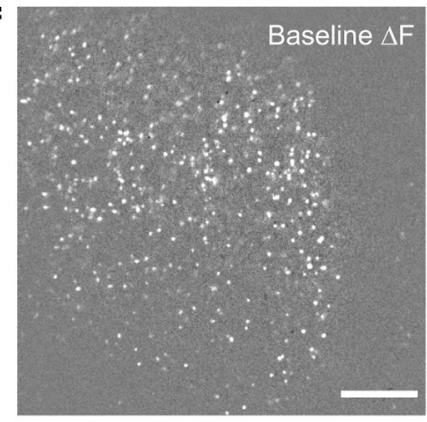

h

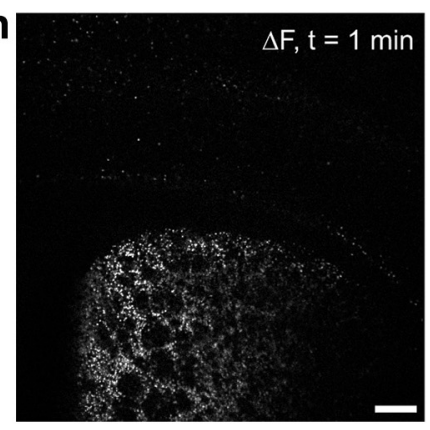

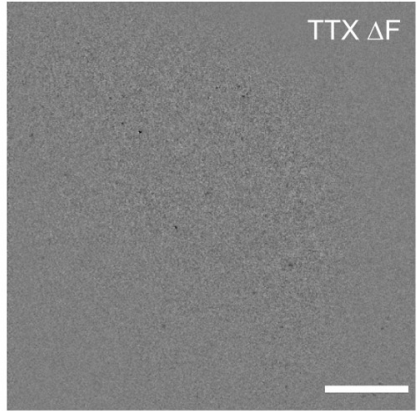

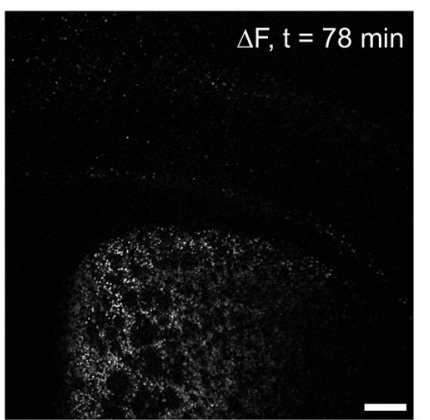

g
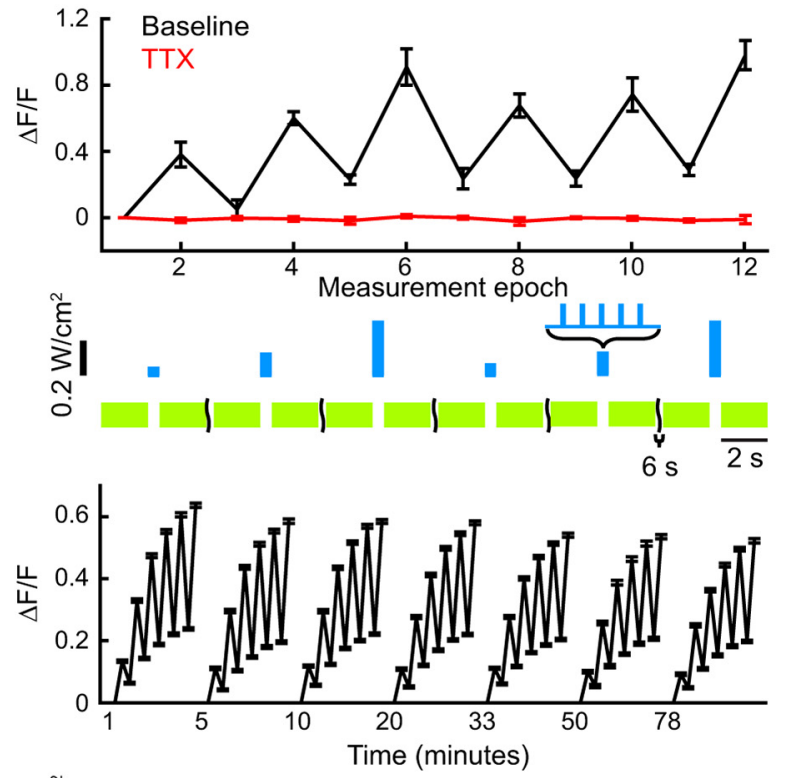

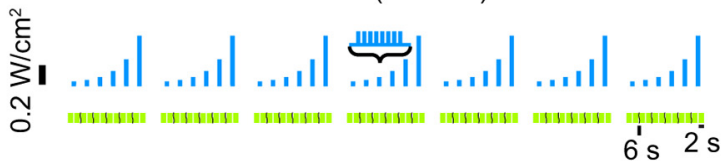

Figure 6. Robust AON in acute brain slices. (a) AAV9 viruses coding for hSyn-eTsChR and hSyn-DO-H2B-jRGECO1a were co-injected in neonatal mouse cortex and striatum. (b) Maximum intensity projection of a Hadamard z-stack of eTsChR expression in a coronal corticostriatal slice from a P21 mouse. (c) Same as (b) in the H2B-jRGEC01a channel. (d) Fluorescence traces from regions indicated in (e). Two cells showed optogenetically induced fluorescence transients, while a region between the cells showed no signal. Here the sets of 11 images acquired before and after each optogenetic stimulus were averaged to form single pre- and post-stimulus fluorescence values. Error bars represent s.e.m. over $n=11$ Hadamard images. Scale bar $25 \mu \mathrm{m}$. Fis defined as the average intensity of the first imaging epoch and $\Delta F$ is the signal increase following blue light stimulation. (e) Magnified view of (c) showing single-cell resolution. ( $f$ ) Mean $\Delta F$ images from striatum before (left) and after (right) addition of TTX (1 $\Delta \mathrm{M}$ ). Images are scaled identically. $(\boldsymbol{g})$ Mean $\Delta F / F$ per measurement epoch from $n=360$ cells in $(\boldsymbol{f})$ before TTX addition (black) and after TTX addition (red). Blue light stimulation consisted of 5 pulses at $12.5 \mathrm{~Hz}$ of $488 \mathrm{~nm}$ light at 60,120 , and $300 \mathrm{~mW} / \mathrm{cm}^{2}$, repeated twice. (h) One slice was repeatedly stimulated and imaged over 78 minutes with protocol in Fig. 3. Mean $\Delta$ Fimages from first run (left) and last run (right), scaled identically. (i) Average $\Delta F / F$ per measurement epoch for $n=3,195$ cells in each run in slice shown in (h). Unless otherwise stated, all scale bars $250 \mu \mathrm{m}$. Error bars indicate \pm SEM.

Probing functional connectivity with ultra-wide-field AON We next sought to extend the Hadamard AON platform to measurements of functional connectivity. Although slicing interrupts many long-range projections, optogenetic stimulation of axon terminals can nonetheless evoke local neurotransmitter release and postsynaptic responses (Petreanu et al., 2007). We reasoned that sufficiently strong presynaptic stimulation would drive postsynaptic spikes, which could be detected via H2B-jRGECO1a.
To achieve this goal, the channelrhodopsin must traffic efficiently to axon terminals. We found that expression of eTsChR was predominantly localized to the soma and dendrites (Fig. 9a). We thus explored CheRiff-TS-GFP (CheRiff), a blue-lightsensitive, high-photocurrent channelrhodopsin (Hochbaum et al., 2014). CheRiff trafficked well in axons (Fig. 9b,c) and was 2.3 -fold more sensitive to blue light than eTsChR. CheRiff was also more sensitive to yellow light, raising the possibility of spurious activation by the $561 \mathrm{~nm}$ imaging laser. Under 


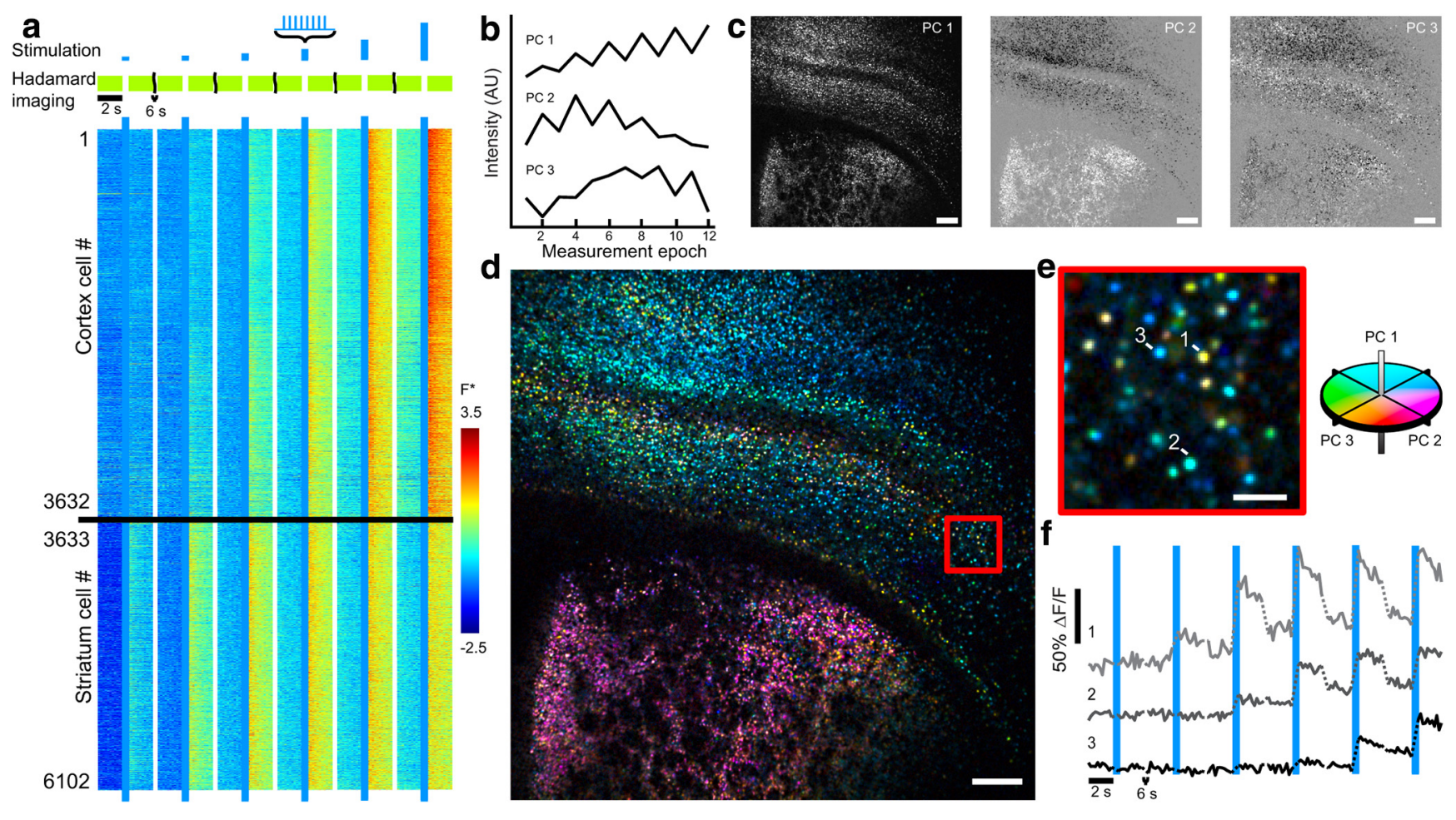

Figure 7. Ultra-wide-field AON in acute brain slices. $\boldsymbol{a}$, Top, Stimulation and imaging protocol. An FOV spanning cortex and striatum was stimulated with eight $5 \mathrm{~ms}$ pulses of $488 \mathrm{~nm}$ light at 20 $\mathrm{Hz}$ with intensities of $15,30,60,120,240$, and $480 \mathrm{~mW} / \mathrm{cm}^{2}$. Bottom, Heat map of 6102 single-cell fluorescence traces acquired simultaneously. Individual fluorescence intensities traces were normalized as $F^{*}=[F-$ mean $(\boldsymbol{F})] / S D(F)$. White breaks separate measurements at different optogenetic stimulus intensities. Imaging was performed at $5.6 \mathrm{~Hz}$ with $100 \mathrm{~mW} / \mathrm{cm}^{2} 561 \mathrm{~nm}$ light. $\boldsymbol{b}$. Waveforms of main principal components from $n=31,754$ cells. See Materials and Methods for additional details. $\boldsymbol{c}$, Principal components from $\boldsymbol{a}$ projected into pixel space. $\boldsymbol{d}$, Image of the slice recorded in $\boldsymbol{a}$ with cells colored by the principal component amplitudes of the functional responses. $\boldsymbol{e}$, Close-up of the red boxed region of $\boldsymbol{d}$. $\boldsymbol{f}$, Three example single-cell fluorescence traces. Dotted lines indicate pauses in Hadamard imaging ( $400 \mathrm{~ms}$ during optogenetic stimulation, $6 \mathrm{~s}$ between stimuli). $\Delta \mathrm{F} / \mathrm{F}$ is defined relative to the intensity in the first imaging epoch. Scale bars: $\boldsymbol{c}, \boldsymbol{d}, 250 \mu \mathrm{m} ; \boldsymbol{e}, 50 \mu \mathrm{m}$.

typical imaging conditions $\left(561 \mathrm{~nm}, 100 \mathrm{~mW} / \mathrm{cm}^{2}\right.$ ) CheRiff photocurrent was $0.9 \%$ of the maximum photocurrent $(95 \%$ confidence interval $0.8-1 \%, n=7$ cells; Fig. $9 d$ ), whereas eTsChR photocurrent was $<0.1 \%$ of its maximum photocurrent (Fig. 2f).

We designed an experiment to express CheRiff in L5 cortico-striatal neurons following a previously described protocol (Wu et al., 2015; Ibrahim et al., 2016), and to test the postsynaptic response via $\mathrm{Ca}^{2+}$ imaging in the striatum. The CheRiff vector comprised CAG-DIO-CheRiff-TS-GFP (Cre-on CheRiff), which we injected into neonatal Rbp4-Cre ${ }^{+/-}$mice to target expression to a population of excitatory L5 neurons. We concurrently injected hSyn-DO-H2B-jRGECO1a (Cre-off nuclear $\mathrm{Ca}^{2+}$ indicator) to drive reporter expression throughout striatum and cortex (Fig. 9e).

First, we tested the slices for spurious activity elicited by the yellow imaging light. Very few striatal neurons showed a detectable increase in H2B-jRGECO1a signal caused by $561 \mathrm{~nm}$ imaging illumination ( $0.32 \pm 0.001 \%, n=3137$ cells, 2 slices; Fig. $9 f, g)$, confirming that the yellow light did not excite axon terminals enough to drive postsynaptic spikes in most cases. This crosstalk performance is not significantly different from that in the eTsChR-based excitability measurements $(0.46 \pm 0.03 \%, n=38,835$ cells, 9 slices, $p=0.25$, two-proportion $z$ test; Fig. 9h). In excitability-style measurements with CheRiff, a significantly larger proportion of neurons showed imaging light-induced activation $(2.3 \pm 0.5 \%, n=944$ cells, 2 slices, $\left.p=8 \times 10^{-10}\right)$. Thus, the superior axonal trafficking of CheRiff made it the preferred actuator for functional connectivity measurements, while the lower yellow-light crosstalk of eTsChR made it the preferred actuator for excitability measurements.
We then repeated the blue-light stimulation and imaging protocol previously used for excitability measurements while monitoring downstream responses in the striatum. Blue light induced nuclear $\mathrm{Ca}^{2+}$ transients across the cortex and striatum (Fig. 9i). Blockers of excitatory transmission, NBQX and CPP, reversibly eliminated the responses in the striatum, L6, and L2/3, confirming that these responses were synaptically evoked (Fig. $9 i$ ) and that there was negligible blue-light crosstalk into the fluorescence signals.

To our surprise, addition of NBQX and CPP reversibly increased the optogenetically induced activity in a population of cells in L5 (Fig. 9j,k). These cells showed little or no response to stimulation before addition of synaptic blockers (Fig. 9k). The location of these cells amid the Rbp4 population suggested that these cells expressed both the actuator and reporter (likely a consequence of imperfect silencing of DO-H2B-jRGECO1a in Rbp4$\mathrm{Cre}^{+}$neurons; Saunders et al., 2012). The increase in excitability upon excitatory blockade then implies a disinhibitory mechanism, i.e., that these $\mathrm{L} 5$ cells received disynaptic inhibition from Rbp4-Cre labeled L5 pyramidal cells, which was relieved under excitatory blockade. The remaining cells in L5 showed a reversible decrease of activity in the presence of excitatory synaptic blockers, similar to the phenotypes in striatum and other cortical layers. These intermixed responses highlight the importance of performing single-cell resolution measurements with Hadamard microscopy. Further, although Hadamard microscopy of jRGECOla can only study suprathreshold responses, these results shown that judicious pharmacological applications can dissect a system's functional connectivity. 


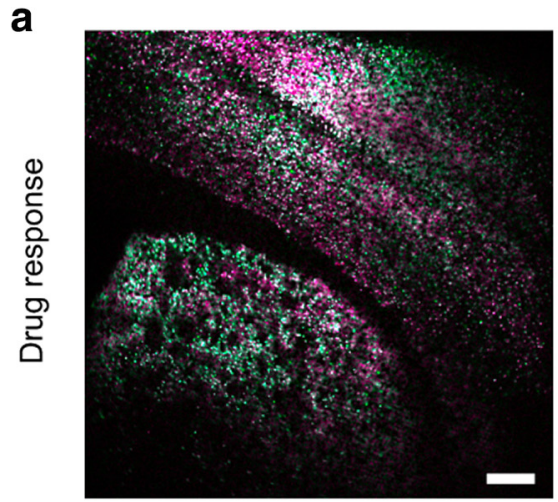

Carbamazepine

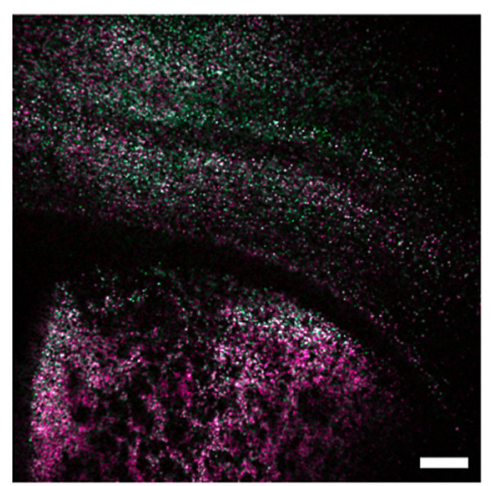

Phenytoin

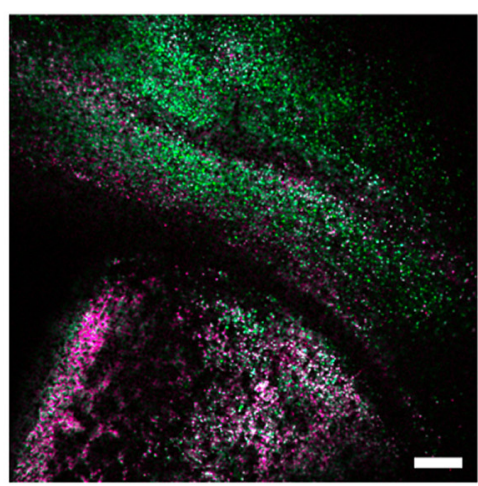

Retigabine

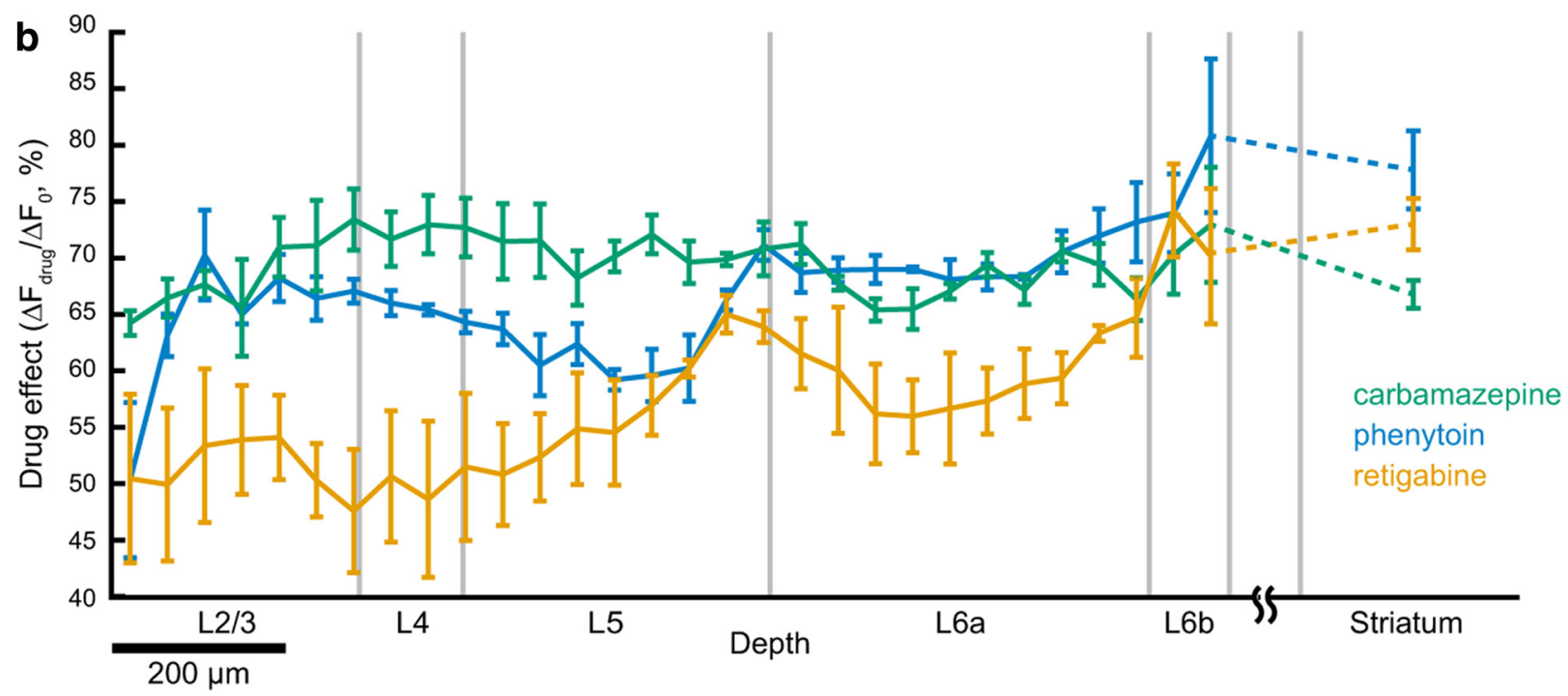

C

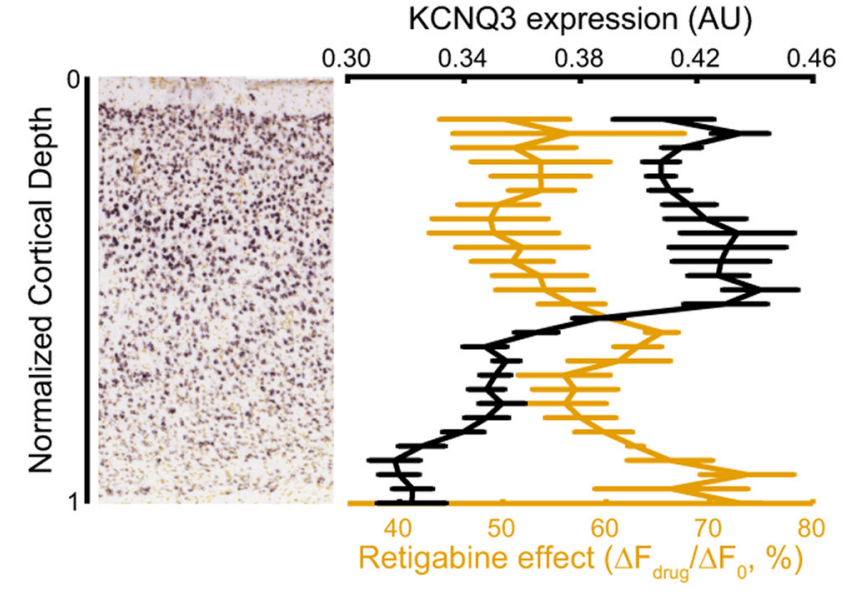

d

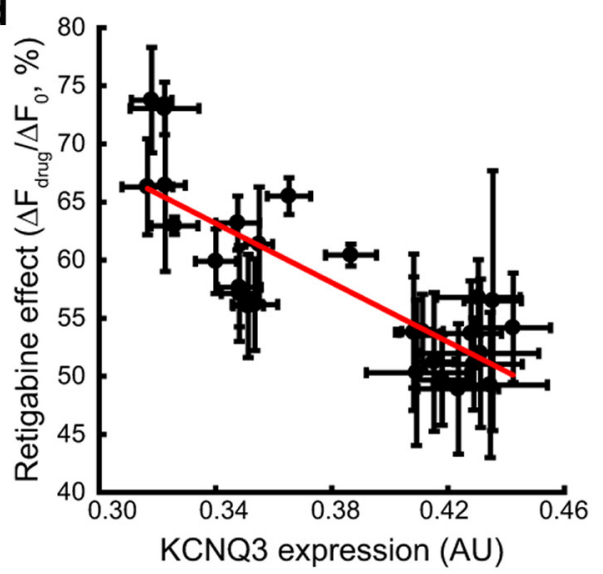

Figure 8. Mapping effects of AEDs on excitability. $\boldsymbol{a}$, Maps of AED effects on excitability. Slices were measured using the excitability protocol as in Figure 7. The protocol was repeated five times before drug addition and four times after addition of carbamazepine (100 $\mu \mathrm{M})$, phenytoin $(100 \mu \mathrm{M})$, or retigabine $(25 \mu \mathrm{m})$. The ratio of mean optogenetically induced change in fluorescence for each cell before $\left(\Delta \mathrm{F}_{0}\right)$ and after drug addition $\left(\Delta \mathrm{F}_{\text {drug }}\right)$ is encoded as color in a green to pink axis. Scale bars, $250 \mu \mathrm{m} . \boldsymbol{b}$, Average drug response $\left(\Delta \mathrm{F}_{\text {drug }} / \Delta \mathrm{F}_{0}\right)$ as a function of cortical depth for $n=3$ slices for each drug. All striatal cells in a slice were pooled into a single bin. Data represent $n=9,793$ cells for carbamazepine, 11,858 cells for phenytoin, and 10,103 cells for retigabine. Error bars represent SEM over $n=3$ slices for each drug. c, Left, In situ hybridization image from Allen Brain Atlas experiment 100041071 showing KCNQ3 expression in somatosensory cortex of a P28 mouse. Right, Cortical depth dependence of retigabine drug effect (same as Fig. 4b) and KCNQ3 expression level determined from in situ hybridization images of $n=11$ slices from the Allen Brain Atlas. $\boldsymbol{d}$, Data from c showing effect of retigabine on excitability versus KCNQ3 expression. Best fit line is indicated in red. Error bars indicate SEM, treating each slice as an independent measurement.

High-speed Hadamard AON with compressed sensing Finally, we illustrate that Hadamard AON can be performed at high speed by using recently developed compressed sensing techniques (Parot et al., 2019). The core idea is to take advantage of the low-rank dynamics of fluorescence fluctuations in neuronal samples. Because each neuron covers multiple camera pixels, every image has redundant information. In the context of a sufficiently long recording, a pair of images comprising a 
a

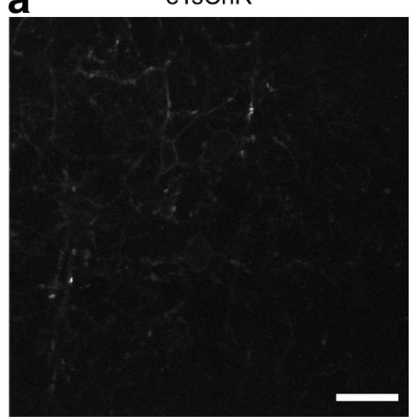

e

CAG-DIO-CheRiff-GFP + hSyn-DO-H2B-jRGECO1a

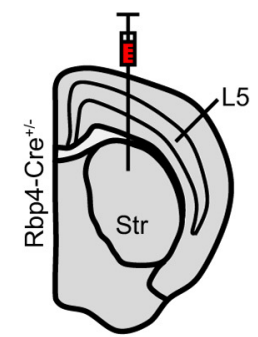

i

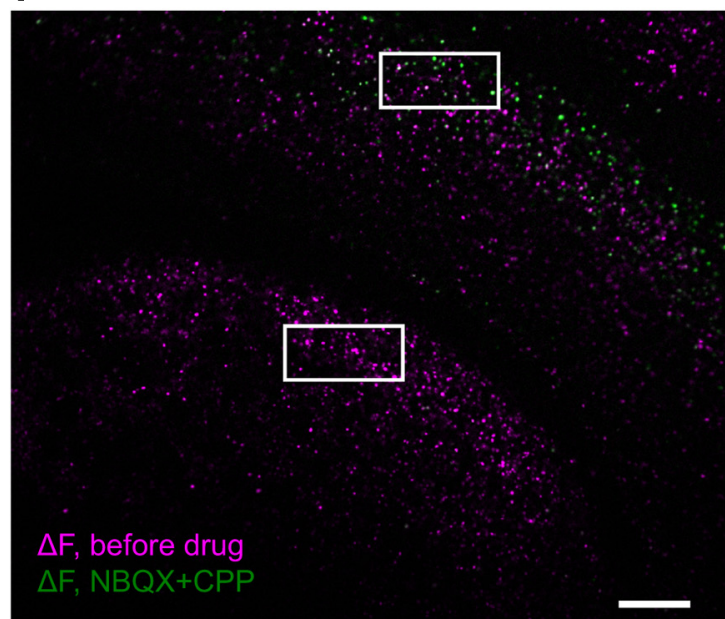

b

CheRiff-TS-GFP
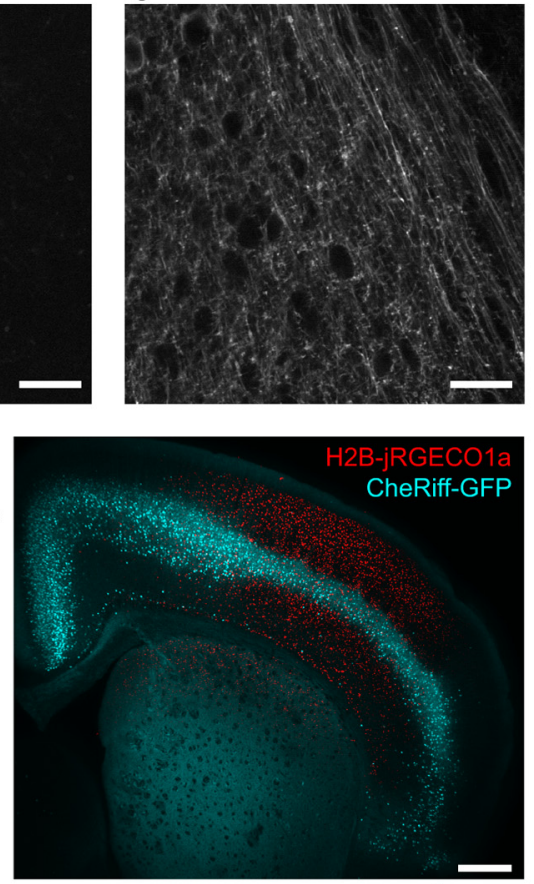

j
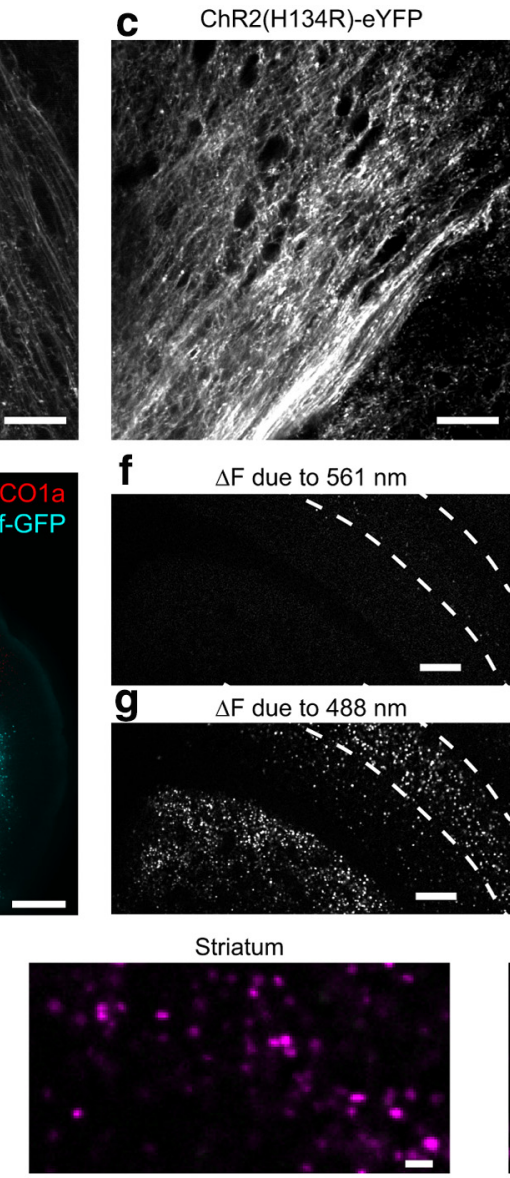

d

f

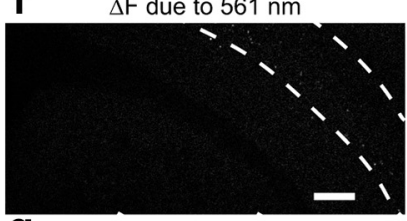

g $\Delta \mathrm{F}$ due to $488 \mathrm{~nm}$

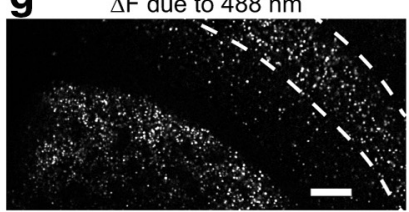

Striatum
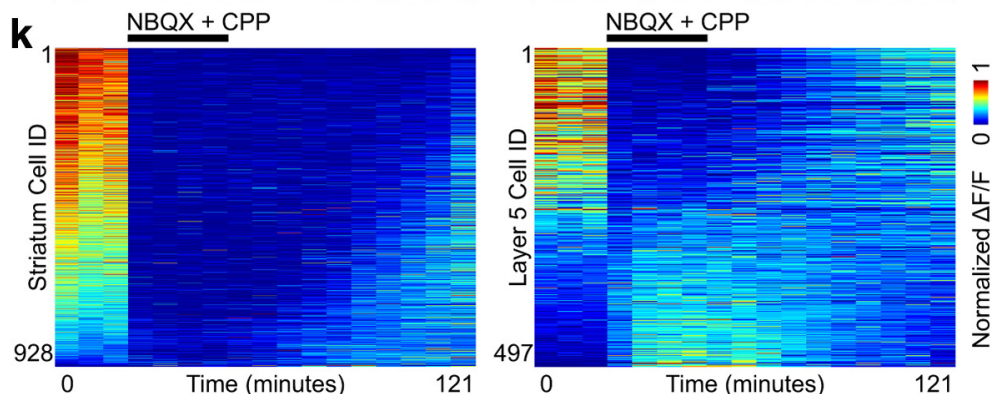

Figure 9. Mapping functional connections. $\boldsymbol{a}-\boldsymbol{c}$, Images of axonal trafficking of eTsChR, CheRiff-TS-GFP, and ChR2(H134R)-YFP, scaled to the same counts. Equal volumes of AAV2/9-hSynChR2(H134R)-eYFP, AAV2/9-hSyn-CheRiff-TS-GFP, and AAV2/9-hSyn-eTsChR were injected in the left hemisphere in separate mice and coronal slices of the contralateral hemisphere were prepared after $>4$ weeks. Images were acquired near the corpus callosum with 2P microscopy. Scale bars: $\boldsymbol{a}-\boldsymbol{c}, 50 \mu \mathrm{m}$. $\boldsymbol{d}$, Comparison of CheRiff photocurrents in HEK293T cells induced by yellow ( $561 \mathrm{~nm}$ ) and blue $(488 \mathrm{~nm})$ light. Vertical bars indicate intensities used in acute slice experiments. The blue illumination intensity to achieve $50 \%$ activation was $94 \mathrm{~mW} / \mathrm{cm}^{2}\left(88,99 \mathrm{~mW} / \mathrm{cm}^{2} 95 \% \mathrm{confidence}\right.$ interval, $n=7$ HEK cells). $\boldsymbol{e}$, Left, Viral constructs for mapping functional connections. Cre-dependent AAV9-CAG-DIO-CheRiff-GFP and AAV9-hSyn-D0-H2B-jRGEC01a were coinjected in Rbp4$\mathrm{Cre}^{+/-}$neonatal mice. Right, At P21, CheRiff-GFP expressed in Cre ${ }^{+} \mathrm{L} 5$ pyramidal cells, including corticostriatal projection neurons. H2B-jRGEC01a expressed broadly in cortex and striatum. Image represents a maximum intensity projection of a Hadamard $z$-stack. Scale bar, $500 \mu \mathrm{m}$. $f$, CheRiff activation by yellow $\left(561 \mathrm{~nm}, 100 \mathrm{~mW} / \mathrm{cm}^{2}\right)$ light. The image shows the difference between mean fluorescence of H2B-jRGECO1a in the second and first second after onset of yellow light for $\mathrm{Ca}^{2+}$ imaging. Image represents a mean of $n=3$ repetitions of the measurement. Spurious CheRiff activation would cause neural firing, which would lead to an increase in H2B-jRGEC01a fluorescence. $\boldsymbol{g}$, Mean $\Delta$ F induced by blue light stimulation, averaged over three runs. $\boldsymbol{g}, \boldsymbol{h}, \boldsymbol{S}$ caled identically. Scale bars: $\boldsymbol{f}, \boldsymbol{g}, 250 \mu \mathrm{m}$. Dashed lines in $\boldsymbol{f}$ and $\boldsymbol{g}$ indicate $L 5$ of the cortex. $\boldsymbol{h}$, Comparison of optical crosstalk for different optogenetic actuators and protocols, as measured by percentage of cells showing $\mathrm{Ca}^{2+}$ transients in response to onset of illumination with $561 \mathrm{~nm}$ light for fluorescence imaging. The three conditions corresponded to eTsChR in the excitability assay (coexpression of actuator and reporter in the same neurons), CheRiff in the excitability assay, and CheRiff in the functional connectivity assay (mutually exclusive expression of actuator and reporter). Error bars are calculated assuming a Poisson distribution with SEM $=\sqrt{n_{\text {active }}} / n_{\text {total }}$. $\boldsymbol{i}$, Mean optogenetically induced fluorescence transients, $\Delta \mathrm{F}$, before (magenta) and after (green) addition of excitatory blockers, NBQX $(10 \mu \mathrm{M})$ and $(\mathrm{PP}(10 \mu \mathrm{M})$. Stimulation and imaging were performed as in Figure 7. Images are the median of three runs before and four runs after adding excitatory blockers. Scale bar, $250 \mu \mathrm{m}$. $\boldsymbol{j}$, Magnified views of indicated regions in striatum and $L 5$ in $\boldsymbol{i}$. Scale bar, $25 \mu \mathrm{m}$. $\boldsymbol{k}$, Mean optogenetically induced fluorescence response, $\Delta \mathrm{F}$, for each cell before, in the presence of, and during washout of excitatory blockers. Left, Striatum; right, L5. Each column represents the mean optogenetically induced $\Delta \mathrm{F}$ of an experimental protocol as in Figure $7 a$. The slice was measured over $121 \mathrm{~min}$ (5-10 min between measurements, 22 min before last measurement). For visualization, each cell trace was normalized by its mean. 

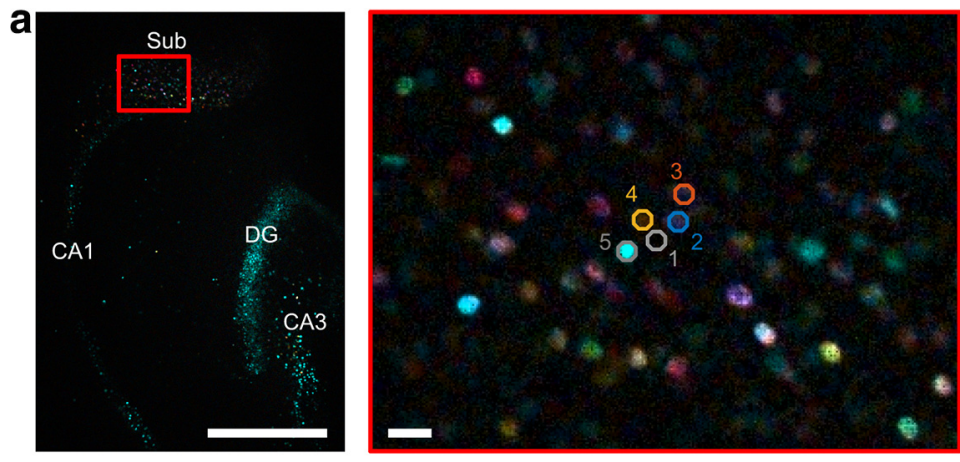

b

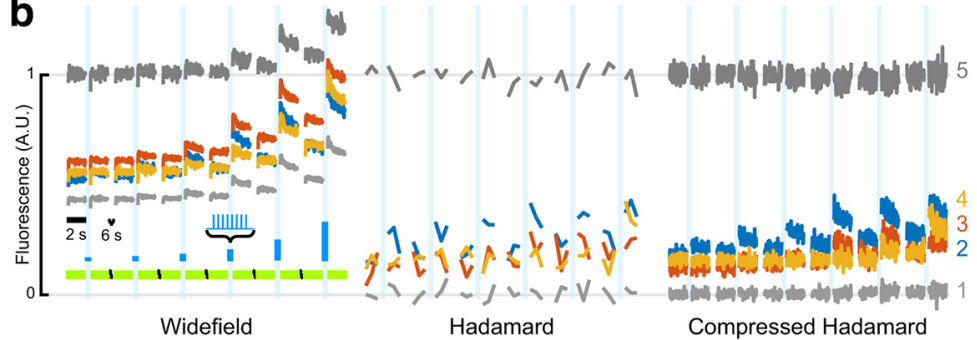

Figure 10. High-speed wide-area all-optical neurophysiology using Compressed Hadamard Imaging. $\boldsymbol{a}$, Left, PCA-colored map showing response of nuclear jRGEC01a to stepped optogenetic stimulation across the hippocampal formation. Sub, Subiculum; DG, dentate gyrus; $\mathrm{CA} 1 / \mathrm{CA}$, cornu ammonis. Scale bar, $0.5 \mathrm{~mm}$. Right, Magnified view of the cyan rectangle shows the location of a background region (1, gray), three responding cells ( $2-4$, colored), and a bright, non-responding cell (5, gray). Scale bar, $20 \mu \mathrm{m}$. Color saturation was increased by $100 \%$ for display. $\boldsymbol{b}$, Comparison of fluorescence traces extracted from Rols in $\boldsymbol{a}$ from the same recording, using three different analyses. Amplitude was scaled to normalize the baseline intensity of the brightest ROI. Left, Wide-field data calculated as the sum of paired complementary patterns. Signals had a time resolution of $33 \mathrm{~Hz}$, but included out-of-focus background. Camera framerate was $66 \mathrm{~Hz}$. Middle, Conventional Hadamard analysis produced one optical section every 48 frames, possibly with errors because of changing cell intensities during the pattern period. Right, Compressed Hadamard analysis obtained high-speed optically sectioned movies with $33 \mathrm{~Hz}$ framerate. Both Hadamard optical sections show a zerocentered value for the background ROI (1) and flat brightness for a non-responding cell (5), but distinct optogenetic activation thresholds for each responding cell (2-4).

single Hadamard pattern and its complement contain enough information to infer the images that would have been recorded under illumination with any other Hadamard pattern. By inferring the complete set of Hadamard images for every pair of camera frames, this approach enables optically sectioned reconstructions at half of the camera frame-rate. Figure 10 illustrates this approach. The sample comprised a hippocampal slice coexpressing eTsChR and nuclear-localized jRGECOla. To ensure that each nucleus was illuminated by multiple DMD pixels, we used a $10 \times$ objective (see Materials and Methods). The optogenetic stimulation was as in Figures 6 and 7. The camera and DMD-patterned illumination were synchronized to run at $66 \mathrm{~Hz}$.

Figure $10 a$ shows a Hadamard image of the whole FOV, in which nuclei have been color-coded by the principal component amplitudes of their dynamic response, as in Figure $7 d$. A close-up image (Fig. 10b) shows the individually resolved nuclei. A widefield image sequence was calculated by averaging together pairs of frames with complementary illumination patterns. Hadamard reconstructions were calculated via the standard approach and via the compressed sensing approach.

In Figure $10 a$, regions 1 and 5 corresponded to a cell-free interstitial region and a non-responsive (presumably dead) very bright cell, respectively, whereas regions $2-4$ corresponded to optogenetically responsive cells. Figure $10 b$ shows that the widefield images had high time resolution, but suffered from out-offocus crosstalk: the gray traces from regions 1 and 5 showed spurious optogenetically induced responses. In the conventional
Hadamard movie, the intensity traces from regions 1 and 5 showed constant fluorescence, confirming effective background rejection, but these extracted traces had low time resolution. In the compressed sensing Hadamard movie, the intensity traces from regions 1 and 5 had the same time resolution as in the wide-field movie $(33 \mathrm{~Hz})$, but lacked background crosstalk as in the conventional Hadamard movie. Thus compressed Hadamard imaging provides a means to achieve simultaneously optical sectioning and high time resolution.

\section{Discussion}

Through detailed photophysical characterization of optogenetic actuators and reporters, we identified pairs that can be used in tandem with minimal $1 \mathrm{P}$ crosstalk. A pairing of CheRiff and jRCaMP1b was recently demonstrated in cultured neurons, but crosstalk was not measured quantitatively and the genetic constructs were not tested in tissue (Afshar Saber et al., 2018). Despite the well reported photophysical blue-light artifacts in jRGECO1a, we found that sufficiently sensitive optogenetic actuators could induce neuronal responses at blue light intensities where these artifacts were minimal. The far blue-shifted channelrhodopsin, eTsChR, enabled measurements of intrinsic excitability, and the highly sensitive channelrhodopsin, CheRiff, enabled measurements of functional connectivity, in both cases with minimal crosstalk from the yellow imaging laser. Finally, nuclear localization of the reporter, combined with Hadamard microscopy enabled resolution of single-cell signals across wide areas of brain slice. The resulting toolbox is well suited for studying suprathreshold phenotypes in acute brain slice, such as excitability of single neurons and functional connectivity of strong excitatory connections. These tools enable wide-area mapping of these properties in brain tissue, and studies on the effects of perturbations thereon.

Questions of where and how neuroactive compounds affect neuronal function are difficult to answer with conventional techniques. Typically, compound distribution is investigated by radiographic labeling experiments. Such results are convolved with possible nonspecific binding of the molecule and with expression of the target in the neuropil, preventing single-cell identification. The $1 \mathrm{P}$ AON technique provides a high spatial resolution functional alternative to radiographic mapping. We show differential response profiles for three AEDs; one molecularly specific drug, retigabine, whose response profile matched its known target distribution; and two nonspecific drugs, carbamazepine and phenytoin. Measurements on other drugs may provide insights into their specific cellular and regional targets. Hadamard AON could also be used to probe the effects of neuropeptides, neuromodulators, hormones, genetic mutations, or environmental perturbations (e.g., temperature, oxygen, metabolites) on brain-wide patterns of neural excitability. 
By extending these assays to measurements of functional connectivity, we show that this $1 \mathrm{P}$ AON toolbox can be also be used for circuit dissection. The all-optical connectivity assay of Figure 9 shows that Rbp4-Cre-positive neurons have a strong excitatory drive across striatum, consistent with previous results (Kozorovitskiy et al., 2015). The net effect of L5 stimulation on other cortical layers was not previously well established; most L5 neurons are excitatory but also recruit strong inhibition via parvalbumin and somatostatin neurons across the cortical column (Jiang et al., 2015; Naka and Adesnik, 2016). We found a clear net excitatory effect of Rbp4-Cre neuron activation in many cells of L2/3 and L6a of the cortex. Within L5 we found a heterogeneous response, where inhibition outweighed excitation in Rbp4-cre-positive neurons (and possibly others which remained nonresponsive during the entire experiment) but excitation outweighed inhibition in other neurons in L5. While this paper was in review, another study interrogated the same circuit with optogenetic stimulation and simultaneous triple whole-cell patch-clamp, with broadly similar conclusions (Adesnik, 2018), though the difficulty of patch-clamp limited the measurements to a few tens of neurons overall.

Both $1 \mathrm{P}$ AON and Hadamard microscopy can be used independently and neither technique is limited to neuroscience applications. The far blue spectrum and excellent sensitivity of eTsChR open the possibility to pair it with red-shifted fluorescent sensors of many other modalities, such as $\mathrm{pH}$, cAMP, or neurotransmitters. The broad spectral range of Hadamard microscopy opens possibilities for high-speed optically sectioned imaging of many different fluorescent reporters, including simultaneous imaging of multiple modalities.

There are many microscopy techniques which could in principle be used for AON in brain slices. Here we briefly outline the factors which led us to develop Hadamard microscopy rather than using an established technique. Spinning disk confocal microscopy (Zeiss; Toomre and Pawley, 2006) in principle provides high temporal resolution and good optical sectioning, but existing spinning disk optics lack sufficient etendue to capture the FOV and NA of the wide-area objective. One could mimic the function of a spinning disk system by activating individual DMD pixels sequentially in a tiled array, acquiring one image per illumination pattern, and then using software spatial filtering to keep only the in-focus component of each point illumination pattern. This approach would yield the same PSF as Hadamard microscopy.

Unmixing techniques such as Hadamard microscopy cannot unmix shot noise. Here we compare the shot noise properties of the Hadamard images relative to DMD-based multifocal confocal. Let $S$ be the mean number of signal photons acquired in one camera pixel in one frame when the corresponding DMD pixel is turned on. Let $\alpha$ be the mean number of background photons acquired in camera pixel $i$ in one frame when DMD pixel $j \neq i$ is turned on, averaged over all $j$ within a block of $N$ pixels. A simple analysis of the shot noise implies that in multifocal confocal (i.e., one DMD pixel turned on at a time) the shot noise-limited signal-to-noise ratio is $S N R=\sqrt{S}$. A short calculation shows that in Hadamard microscopy, the shot noiselimited SNR is approximately:

$$
S N R=\frac{S}{\sqrt{2 \alpha}} .
$$

If $\alpha<S / 2$, then Hadamard is better; otherwise multifocal confocal is better. We found experimentally that for $N=12, \alpha / S=$
0.51 , indicating comparable shot noise for the multifocal and Hadamard approaches. For $N=64$, we measured $\alpha / S=0.26$, indicating superior performance of Hadamard over multifocal confocal. In these comparisons, the total optical dose into the sample is greater for Hadamard than for multifocal. The duty cycle of illumination is $1 / N$ for multifocal confocal, and $1 / 2$ for Hadamard, so for $N=12$, Hadamard exposes the sample to sixfold more light, and for $N=64$, Hadamard uses 32-fold more light. Although photobleaching and phototoxicity were not significant factors in the present experiments, the presence of these effects may favor multifocal confocal.

Stripe SIM and HiLo techniques are alternatives that could be implemented with the same DMD optics as Hadamard microscopy. The improved PSF shape (relative to stripe SIM and HiLo) and the absence of static illumination noise (relative to HiLo) favored Hadamard microscopy. The contributions of background photons to the shot noise are identical in all three techniques. The lower temporal resolution of Hadamard relative to the other SIM techniques did not constrain the ability to map nuclear $\mathrm{Ca}^{2+}$ dynamics, though better time resolution may be needed for other fluorescent reporters. Improvement in the time resolution of Hadamard microscopy are possible via compressed sensing techniques (Parot et al., 2019).

2P mesoscopes currently hold the record for most single neurons $(\sim 3000)$ recorded simultaneously in tissue (Sofroniew et al., 2016). 2P mesoscopes have greater depth penetration than SIM techniques, making them more suitable for in vivo studies at present. Point-scanning based mesoscopes have achieved pixel rates of $\sim 2 \times 10^{7} / \mathrm{s}$ over $0.6 \times 0.6 \mathrm{~mm}$ FOVs but the requirement to translate the beam long distances limits pixel rates over large FOVs $(4.4 \times 4.2 \mathrm{~mm})$ to $5.6 \times 10^{6} / \mathrm{s}$. Acoustooptical steering allows fast $2 \mathrm{P}$ random-access imaging (Szalay et al., 2016), but this technique has only been demonstrated in a FOV of $0.5 \mathrm{~mm}$, limited by the etendue of the acoustooptical deflectors. With 12-pattern Hadamard, we achieved comparable data rates of $1.2 \times 10^{7} / \mathrm{s}$ over a $4.6 \times 2.3 \mathrm{~mm}$ FOV, with optically sectioned single-cell resolution. With improved control software to synchronize Hadamard patterns to the rolling shutter of the camera, pixel rates of $3.3 \times 10^{7}$ pixels $/ \mathrm{s}$ over the entire $4.6 \times 4.6 \mathrm{~mm}$ FOV would be possible with current camera technology. Finally, in contrast to $2 \mathrm{P}$ mesoscopes, Hadamard microscopy is readily implemented with inexpensive LED or diode laser illumination across a broad range of excitation wavelengths.

Even when imaging in acute slices, one must achieve sufficient depth penetration to avoid damaged cells near the surface. Because of the great effort and low throughput of manual patchclamp measurements, it is typical to record at a depth of $50 \mu \mathrm{m}$ or greater to minimize the risk of patching an unhealthy cell, though recent protocols suggest $30 \mu \mathrm{m}$ is sufficient (Ting et al., 2018). Our approach includes procedures to identify and exclude dead (nonresponsive) cells. Hadamard AON measures large enough numbers of cells that one can afford to discard a few unhealthy cells, while still maintaining excellent statistics. The stable excitability and anticipated responses to tool pharmacology (Fig. 6) establish that the cells assayed in our methods are adequately healthy from a functional perspective, despite being only $\sim 30$ $\mu \mathrm{m}$ below the surface.

For precisely targeted single-cell stimulation, $2 \mathrm{P}$ optics are essential, but for wide-area optogenetic stimulation, $1 \mathrm{P}$ optics are preferable, as follows: $2 \mathrm{P}$ optogenetic stimulation requires time-average optical powers of 20-80 mW/cell (Ronzitti et al., 2017; Shemesh et al., 2017; Mardinly et al., 2018). Maximal safe steady-state 2P optical 
power into intact brain tissue is $\sim 200 \mathrm{~mW}$ (Podgorski and Ranganathan, 2016), limiting simultaneous $2 \mathrm{P}$ stimulation to at most a few tens of neurons at a time. $1 \mathrm{P}$ optogenetic stimulation requires $\sim 10^{6}$ fold lower time-average power ( $\sim 50 \mathrm{nW} /$ cell; Hochbaum et al., 2014), and thus is readily applied over wide areas of tissue to many thousands of cells simultaneously.

If a microscope can measure $N$ cells in parallel, a measurement protocol takes time $\tau$, and an acute slice is viable for time $T$, then the total number of cells that can be measured is $N T / \tau$. A typical excitability measurement (including focusing and saving data) takes $\tau \sim 2 \mathrm{~min}$. In the present work, the FOV was limited by the range of viral gene expression, but in transgenic animals or with recently developed systemic gene delivery techniques (Chan et al., 2017), functional measurements could be made across an entire brain slice. With the current FOV, Hadamard microscopy could tile a complete sagittal slice in seven acquisitions, or $\sim 14 \mathrm{~min}$. Brain slices typically remain viable for $T \sim 5 \mathrm{~h}$. Thus one could record from $\sim 20$ slices, enough to create a brain-wide functional map. Such a mapping technique could provide an unbiased approach to studying neuronal excitability, functional connectivity, and pharmacology across an entire brain.

\section{References}

Adesnik H (2018) Layer-specific excitation/inhibition balances during neuronal synchronization in the visual cortex. J Physiol 596:1639-1657.

Afshar Saber W, Gasparoli FM, Dirks MG, Gunn-Moore FJ, Antkowiak M (2018) All-optical assay to study biological neural networks. Front Neurosci 12:451.

Akerboom J, Carreras Calderón N, Tian L, Wabnig S, Prigge M, Tolö J, Gordus A, Orger MB, Severi KE, Macklin JJ, Patel R, Pulver SR, Wardill TJ, Fischer E, Schüler C, Chen TW, Sarkisyan KS, Marvin JS, Bargmann CI, Kim DS, et al. (2013) Genetically encoded calcium indicators for multi-color neural activity imaging and combination with optogenetics. Front Mol Neurosci 6:2.

Bengtson CP, Freitag HE, Weislogel JM, Bading H (2010) Nuclear calcium sensors reveal that repetition of trains of synaptic stimuli boosts nuclear calcium signaling in CA1 pyramidal neurons. Biophys J 99:40664077.

Blot A, Barbour B (2014) Ultra-rapid axon-axon ephaptic inhibition of cerebellar purkinje cells by the pinceau. Nat Neurosci 17:289-295.

Carrillo-Reid L, Yang W, Bando Y, Peterka DS, Yuste R (2016) Imprinting and recalling cortical ensembles. Science 353:691-694.

Chan KY, Jang MJ, Yoo BB, Greenbaum A, Ravi N, Wu WL, SánchezGuardado L, Lois C, Mazmanian SK, Deverman BE, Gradinaru V (2017) Engineered AAVs for efficient noninvasive gene delivery to the central and peripheral nervous systems. Nat Neurosci 20:1172-1179.

Chen TW, Wardill TJ, Sun Y, Pulver SR, Renninger SL, Baohan A, Schreiter ER, Kerr Ra, Orger MB, Jayaraman V, Looger LL, Svoboda K, Kim DS (2013) Ultrasensitive fluorescent proteins for imaging neuronal activity. Nature 499:295-300.

Dana H, Mohar B, Sun Y, Narayan S, Gordus A, Hasseman JP, Tsegaye G, Holt GT, Hu A, Walpita D, Patel R, Macklin JJ, Bargmann CI, Ahrens MB, Schreiter ER, Jayaraman V, Looger LL, Svoboda K, Kim DS (2016) Sensitive red protein calcium indicators for imaging neural activity. eLife 5:e12727.

Eder A, Bading H (2007) Calcium signals can freely cross the nuclear envelope in hippocampal neurons: somatic calcium increases generate nuclear calcium transients. BMC Neurosci 8:57.

Emiliani V, Cohen AE, Deisseroth K, Häusser M (2015) All-optical interrogation of neural circuits. J Neurosci 35:13917-13926.

Freeman J, Vladimirov N, Kawashima T, Mu Y, Sofroniew NJ, Bennett DV, Rosen J, Yang CT, Looger LL, Ahrens MB (2014) Mapping brain activity at scale with cluster computing. Nat Methods 11:941-950.

Gunthorpe MJ, Large CH, Sankar R (2012) The mechanism of action of retigabine (ezogabine), a first-in-class $\mathrm{K}+$ channel opener for the treatment of epilepsy. Epilepsia 53:412-424.

Gustafsson MG (2000) Surpassing the lateral resolution limit by a factor of two using structured illumination microscopy. J Microsc 198:82-87.
Harnett MT, Xu NL, Magee JC, Williams SR (2013) Potassium channels control the interaction between active dendritic integration compartments in layer 5 cortical pyramidal neurons. Neuron 79:516-529.

Hochbaum DR, Zhao Y, Farhi SL, Klapoetke N, Werley CA, Kapoor V, Zou P, Kralj JM, Maclaurin D, Smedemark-Margulies N, Saulnier JL, Boulting GL, Straub C, Cho YK, Melkonian M, Wong GK, Harrison DJ, Murthy VN, Sabatini BL, Boyden ES, et al. (2014) All-optical electrophysiology in mammalian neurons using engineered microbial rhodopsins. Nat Methods 11:825-833.

Huang YL, Walker AS, Miller EW (2015) A photostable silicon rhodamine platform for optical voltage sensing. J Am Chem Soc 137:10767-10776.

Ibrahim LA, Mesik L, Ji XY, Fang Q, Li HF, Li YT, Zingg B, Zhang LI, Tao HW (2016) Cross-modality sharpening of visual cortical processing through layer-1-mediated inhibition and disinhibition. Neuron 89:1031-1045.

Inoue M, Takeuchi A, Horigane S, Ohkura M, Gengyo-Ando K, Fujii H, Kamijo S, Takemoto-Kimura S, Kano M, Nakai J, Kitamura K, Bito H (2015) Rational design of a high-affinity, fast, red calcium indicator R-CaMP2. Nat Methods 12:64-70.

Jiang X, Shen S, Cadwell CR, Berens P, Sinz F, Ecker AS, Patel S, Tolias AS (2015) Principles of connectivity among morphologically defined cell types in adult neocortex. Science 350:aac9462.

Klapoetke NC, Murata Y, Kim SS, Pulver SR, Birdsey-Benson A, Cho YK, Morimoto TK, Chuong AS, Carpenter EJ, Tian Z, Wang J, Xie Y, Yan Z, Zhang Y, Chow BY, Surek B, Melkonian M, Jayaraman V, ConstantinePaton M, Wong GK, et al. (2014) Independent optical excitation of distinct neural populations. Nat Methods 11:338-346.

Klein S, Staring M, Murphy K, Viergever MA, Pluim JP (2010) Elastix: a toolbox for intensity-based medical image registration. IEEE Trans Med Imaging 29:196-205.

Kozorovitskiy Y, Peixoto R, Wang W, Saunders A, Sabatini BL (2015) Neuromodulation of excitatory synaptogenesis in striatal development. eLife 4:e10111.

Lein ES, Hawrylycz MJ, Ao N, Ayres M, Bensinger A, Bernard A, Boe AF, Boguski MS, Brockway KS, Byrnes EJ, Chen L, Chen L, Chen TM, Chin MC, Chong J, Crook BE, Czaplinska A, Dang CN, Datta S, Dee NR, et al. (2007) Genome-wide atlas of gene expression in the adult mouse brain. Nature 445:168-176.

Mardinly AR, Oldenburg IA, Pégard NC, Sridharan S, Lyall EH, Chesnov K, Brohawn SG, Waller L, Adesnik H (2018) Precise multimodal optical control of neural ensemble activity. Nat Neurosci 21:881-893.

Mazzaferri J, Kunik D, Belisle JM, Singh K, Lefrançois S, Costantino S (2011) Analyzing speckle contrast for HiLo microscopy optimization. Optics Express 19:14508-14517.

Mertz J (2011) Optical sectioning microscopy with planar or structured illumination. Nat Methods 8:811-819.

Mertz J, Kim J (2010) Scanning light-sheet microscopy in the whole mouse brain with HiLo background rejection. J Biomed Opt 15:016027.

Naka A, Adesnik H (2016) Inhibitory circuits in cortical layer 5. Front Neural Circuits 10:35.

Packer AM, Russell LE, Dalgleish HW, Häusser M (2015) Simultaneous alloptical manipulation and recording of neural circuit activity with cellular resolution in vivo. Nat Methods 12:140-146.

Parot VJ, Sing-Long C, Adam Y, Boehm UL, Fan L, Farhi SL, Cohen AE (2019) Compressed hadamard microscopy for high-speed optically sectioned neuronal activity recordings. J Phys D Appl Phys 52:144001.

Petreanu L, Huber D, Sobczyk A, Svoboda K (2007) Channelrhodopsin-2assisted circuit mapping of long-range callosal projections. Nat Neurosci 10:663-668.

Podgorski K, Ranganathan G (2016) Brain heating induced by near-infrared lasers during multiphoton microscopy. J Neurophysiol 116:1012-1023.

Rickgauer JP, Deisseroth K, Tank DW (2014) Simultaneous cellularresolution optical perturbation and imaging of place cell firing fields. Nat Neurosci 17:1816-1824.

Ronzitti E, Ventalon C, Canepari M, Forget BC, Papagiakoumou E, Emiliani V (2017) Recent advances in patterned photostimulation for optogenetics. J Optics 19:113001.

Saganich MJ, Machado E, Rudy B (2001) Differential expression of genes encoding subthreshold-operating voltage-gated $\mathrm{K}+$ channels in brain. J Neurosci 21:4609-4624.

Saunders A, Johnson CA, Sabatini BL (2012) Novel recombinant adeno- 
associated viruses for Cre activated and inactivated transgene expression in neurons. Front Neural Circuits 6:47.

Shemesh OA, Tanese D, Zampini V, Linghu C, Piatkevich K, Ronzitti E, Papagiakoumou E, Boyden ES, Emiliani V (2017) Temporally precise single-cell-resolution optogenetics. Nat Neurosci 20:1796-1806.

Skocek O, Nöbauer T, Weilguny L, Martínez Traub F, Xia CN, Molodtsov MI, Grama A, Yamagata M, Aharoni D, Cox DD, Golshani P, Vaziri A (2018) High-speed volumetric imaging of neuronal activity in freely moving rodents. Nat Methods 15:429-432.

Sofroniew NJ, Flickinger D, King J, Svoboda K (2016) A large field of view two-photon mesoscope with subcellular resolution for in vivo imaging. eLife 5:e14472.
Szalay G, Judák L, Katona G, Ócsai K, Juhász G, Veress M, Szadai Z, Fehér A, Tompa T, Chiovini B, Maák P, Rózsa B (2016) Fast 3D imaging of spine, dendritic, and neuronal assemblies in behaving animals. Neuron 92:723-738.

Ting JT, Lee BR, Chong P, Soler-Llavina G, Cobbs C, Koch C, Zeng H, Lein E (2018) Preparation of acute brain slices using an optimized N-methyl-Dglucamine protective recovery method. J Vis Exp 132:e53825.

Toomre D, Pawley JB (2006) Disk-scanning confocal microscopy. Handbook of biological confocal microscopy, pp 221-238. Boston, MA:Springer.

Wu YW, Kim JI, Tawfik VL, Lalchandani RR, Scherrer G, Ding JB (2015) Input- and cell-type-specific endocannabinoid-dependent LTD in the striatum. Cell Rep 10:75-87. 